\title{
Galaxy stellar mass assembly: the difficulty matching observations and semi-analytical predictions ${ }^{\star}$
}

\author{
M. Cousin ${ }^{1}$, G. Lagache ${ }^{1,5}$, M. Bethermin ${ }^{4}$, J. Blaizot ${ }^{2,3}$, and B. Guiderdoni ${ }^{2,3}$ \\ ${ }^{1}$ Institut d'Astrophysique Spatiale (IAS), Bâtiment 121, Université Paris-Sud 11 and CNRS (UMR 8617), 91405 Orsay, France \\ e-mail: morgane.cousin@ias.u-psud.fr \\ 2 Université Lyon 1, Observatoire de Lyon, 9 avenue Charles André, 69230 Saint-Genis-Laval, France \\ 3 CNRS (UMR 5574), Centre de Recherche Astrophysique de Lyon, École Normale Supérieure de Lyon, 69007 Lyon, France \\ ${ }^{4}$ European Southern Observatory, Karl-Schwarzschild-Str. 2, 85748 Garching, Germany \\ 5 Aix Marseille Université, CNRS, LAM (Laboratoire d'Astrophysique de Marseille) UMR 7326, 13388 Marseille, France
}

Received 15 November 2013 / Accepted 6 October 2014

\begin{abstract}
Semi-analytical models (SAMs) are currently the best way to understand the formation of galaxies within the cosmic dark-matter structures. They are able to give a statistical view of the variety of the evolutionary histories of galaxies in terms of star formation and stellar mass assembly. While they reproduce the local stellar mass functions, correlation functions, and luminosity functions fairly well, they fail to match observations at high redshift $(z \geq 3)$ in most cases, particularly in the low-mass range. The inconsistency between models and CDM observations indicates that the history of gas accretion in galaxies, within their host dark-matter halo, and the transformation of gas into stars, are not followed well. We briefly present a new version of the GalICS semi-analytical model. With this new model, we explore the impact of classical mechanisms, such as supernova feedback or photoionization, on the evolution of the stellar mass assembly and the star formation rate. Even with strong efficiency, these two processes cannot explain the observed stellar mass function and star formation rate distribution or the stellar mass versus dark matter halo mass relation. We thus introduce an ad hoc modification of the standard paradigm, based on the presence of a no-star-forming gas component, and a concentration of the star-forming gas in galaxy discs. The main idea behind the existence of the no-star-forming gas reservoir is that only a fraction of the total gas mass in a galaxy is available to form stars. The reservoir generates a delay between the accretion of the gas and the star formation process. This new model is in much better agreement with the observations of the stellar mass function in the low-mass range than the previous models and agrees quite well with a large set of observations, including the redshift evolution of the specific star formation rate. However, it predicts a large amount of no-star-forming baryonic gas, potentially larger than observed, even if its nature has still to be examined in the context of the missing baryon problem. Outputs from all models are available at the CDS.
\end{abstract}

Key words. galaxies: formation - galaxies: evolution - galaxies: star formation - galaxies: halos

\section{Introduction}

Cosmological models based on the $\Lambda$-cold-dark-matter (CDM) paradigm have proved remarkably successful at explaining the origin and evolution of structures in the Universe. Since the pioneer work of Blumenthal et al. (1984), this model has become a powerful tool for describing the evolution of primordial density fluctuations leading to the large scale structures (e.g. Peacock et al. 2001; Spergel et al. 2003; Planck Collaboration XVI 2014). Galaxy clustering or weak gravitational lensing are modelled very well in this framework (e.g. Fu et al. 2008).

The description of smaller scales (galaxies) is more problematic. Even if we put aside the problem of the angular momentum transfer between the disc and the dark-matter host halo, there are still some challenges on sub-galaxy scales. Twenty years ago, Kauffmann et al. (1993) pointed out the so-called sub-structure problem. Indeed the large amount of power on small scales in the $\Lambda$-CDM paradigm generates an over-estimate of the number of small objects (with properties close to dwarf galaxies). The overdensity of substructures is clearly seen in $N$-body simulations at

\footnotetext{
* Outputs from all models are only available at the CDS via anonymous ftp to cdsarc.u-strasbg. fr (130.79.128.5) or via http://cdsarc.u-strasbg.fr/viz-bin/qcat?]/A+A/575/A32
}

low redshift $(z \simeq 0)$. Dark matter haloes with mass comparable to that of our Galaxy $\left(M_{\mathrm{h}} \simeq 10^{12} M_{\odot}\right)$ contain more than one hundred substructures enclosed in their virial radius. In contrast, the observations of the Local Group count fifty satellite galaxies at most.

This effect is even more problematic at high redshift $(z>1)$. Indeed, coupled with the poor understanding of the star formation process in these small haloes, the standard scenario produces a large excess of stellar mass in low-mass structures (Guo et al. 2011). To limit the number of dwarf galaxies, galaxy formation models, such as semi-analytical model (SAM) or cosmological hydrodynamic simulations, invoke gas photoionization and strong supernova feedback (Efstathiou 1992; Shapiro et al. 1994; Babul \& Rees 1992; Quinn et al. 1996; Thoul \& Weinberg 1996; Bullock et al. 2000; Gnedin 2000; Benson et al. 2002; Somerville 2002; Croton et al. 2006; Hoeft et al. 2006; Okamoto et al. 2008; Somerville et al. 2008, 2012). Originally proposed by Doroshkevich et al. (1967), photoionization has been developed in the CDM paradigm by Couchman \& Rees (1986), Ikeuchi (1986), and Rees (1986). The idea is quite simple: the ultraviolet (UV) background generated by the quasars and first generations of stars heats the gas. In the small structures, the temperature reached by the gas is then too high, preventing it from collapsing 
into dark matter haloes. The accretion of the gas on the galaxies, hence the star formation, is thus reduced.

Many semi-analytical models strive to reproduce the luminous properties of galaxy samples, such as luminosity functions or galaxy number counts (Cole et al. 2000; Croton et al. 2006; Hatton et al. 2003; Monaco et al. 2007; Somerville et al. 2008). This approach has to be linked to the nature of the observational constraints. Indeed, ten years ago, broad-band luminosity measurements were the main constraints. In general, local luminosity functions in the optical domain were well reproduced by standard SAMs (Cole et al. 2000; Hatton et al. 2003; Croton et al. 2006; Baugh 2006; Guo et al. 2011). But first analysis including the dust reprocessing showed a deep misunderstanding of the star formation processes (Granato et al. 2000). Study of the cosmic infrared background, added to UV and optical measurements, indicates a peak of star formation activity for $1<z<4$ (Lilly et al. 1996; Madau et al. 1996; Gispert et al. 2000; Chary \& Elbaz 2001; Le Floc'h et al. 2005; Hopkins \& Beacom 2006; Dunne et al. 2009; Rodighiero et al. 2010; Gruppioni et al. 2010). The star formation rate distribution (or IR luminosity function IR-LF) of galaxies at these epochs is currently not reproduced well by the physical models (Bell et al. 2007; Le Floc'h et al. 2009; Rodighiero et al. 2010; Magnelli et al. 2011). Also discrepancies between models and observations are large for the galaxy number counts at long wavelengths $(\lambda>100 \mu \mathrm{m})$ or the redshift distributions of star-forming galaxies (Hatton et al. 2003; Baugh 2006; Somerville et al. 2012). Prescriptions were developed to try to reduce the discrepancy, such as the modification of the initial mass function (IMF) in starbursts (Guiderdoni et al. 1997; Baugh 2006). Even if thus a modification improves the galaxy number counts in the farinfrared wavelengths there is no observational evidence of such an IMF variation.

Today, with the new observational constraints, such as those derived from galaxy-galaxy lensing (McKay et al. 2001; Hoekstra et al. 2004; Mandelbaum et al. 2006a,b; Leauthaud et al. 2010), we have access to more fundamental galaxy properties: stellar mass $M_{\star}$, star formation rates (SFR), and to the links between them (Brinchmann et al. 2004; Noeske et al. 2007; Dunne et al. 2009; Elbaz et al. 2011; Karim et al. 2011). With the development of new techniques, such as the abundance matching, the relation between stellar mass, galaxy mass, dark matter halo mass, or even between SFR and $M_{\mathrm{h}}$ can be explored (Conroy \& Wechsler 2009; Béthermin et al. 2012; Behroozi et al. 2013a). Consequently, SAMs added some other relations to the analysis of the luminous properties of galaxies, such as the specific star formation rate $\left(S S F R=S F R / M_{\star}\right)$ and its redshift evolution, or the stellar mass $\left(M_{\star}\right)$ versus dark matter halo mass $\left(M_{\mathrm{h}}\right)$ relation (SHMR; Guo et al. 2011; Leauthaud et al. 2012). The work presented here continues this effort.

In this paper, we used a new semi-analytical model (detailed in Cousin et al. 2015) built on recent theoretical prescriptions and hydrodynamic simulation results (Bertone et al. 2005; Kereš et al. 2005; Bournaud et al. 2007; Genzel et al. 2008; Dekel et al. 2009a,b; Khochfar \& Silk 2009; van de Voort et al. 2011; Faucher-Giguère et al. 2011; Lu et al. 2011; Capelo et al. 2012), and we compare it to an up-to-date set of observations. Our goal is to better understand the model parameters (physical recipes) that have to be strongly modified to obtain good agreement between models and observations.

The paper is organized as follows. In Sect. 2, we describe the main features of our SAM. In Sect. 3, we explore the impact of classical photoionization and supernova (SN)-feedback recipes on fundamental galaxy properties: stellar mass function
(SMF), $M_{\mathrm{h}}$ versus $M_{\star}$ relation (SHMR), and specific star formation rate (sSFR). We add to these properties the SFR distribution (or the IR-LF) and its redshift evolution. We show that the basic models fail to reproduce these kinds of measurements and propose the existence of a no-star-forming gas reservoir in galaxy discs to reconcile the models with the observations (Sect. 4). We present a detailed comparison between models and observations in Sect. 5. We conclude in Sect. 6. Throughout the paper we use Chabrier (2003) initial mass function (IMF).

\section{Brief description of the model}

The SAM briefly presented here is a revised version of the GalICS model (Hatton et al. 2003). We did a detailed analysis of the dark-matter merger tree properties and have revised the description of baryonic physics using the most recent prescriptions extracted from analytical works and hydrodynamic simulations. A complete description is provided in a companion paper (Cousin et al. 2015, towards a new modelling of gas flows in a semi-analytical model of galaxy formation and evolution).

\subsection{Dark matter}

Like its predecessor, our model is based on a hybrid approach. We use dark-matter merger trees extracted from a pure $N$-body simulation. This simulation, with WMAP-3yr cosmology $\left(\Omega_{\mathrm{m}}=\right.$ $\left.0.24, \Omega_{\Lambda}=0.76, f_{\mathrm{b}}=0.16, h=0.73\right)$, describes a volume of $\left(100 h^{-1}\right)^{3} \simeq 150 \mathrm{Mpc}^{3}$. In this volume, $1024^{3}$ particles evolve with an elementary mass of $m_{\mathrm{p}}=8.536 \times 10^{7} M_{\odot}$. We use the HaloMaker code described in Tweed et al. (2009) to identify the haloes and their sub-structures, and build merger trees. We only consider dark-matter structures containing at least 20 darkmatter particles. This limit gives a minimal dark matter halo mass $M_{\mathrm{h}}^{\min }=1.707 \times 10^{9} M_{\odot}$.

In addition to the merger-tree building, we have added a posttreatment to the dark-matter haloes. Based on the time-integrated halo mass and on the energy and halo spin parameter evolution, we selected the healthy population of haloes, i.e., haloes with a negative total gravitational energy and a smooth evolution of the spin parameter. The tree branches that do not satisfy the conditions are considered as smooth accretion $(\simeq 1-5 \%$ of the total mass identified in haloes at a given time). There are no galaxies in these kinds of tree branches.

\subsection{Adding baryons}

In hybrid SAMs, the baryonic physics are added to the preevolved dark-matter background. The baryonic mass is added progressively, following the dark-matter smooth accretion: $\dot{M}_{\mathrm{b}}=$ $f_{\mathrm{b}}^{\mathrm{ph}-\text { ion }}\left(M_{\mathrm{h}}, z\right) \dot{M}_{\mathrm{dm}}$, where $f_{\mathrm{b}}^{\mathrm{ph}-\text { ion }}\left(M_{\mathrm{h}}, z\right)$ depends on the photoionization model. In our case, we use the Okamoto et al. (2008) prescription in the reference model $m_{1}$ (see Sect. 3.1 for more information), and we use Gnedin (2000) as a model variation.

In the current galaxy formation paradigm, the baryonic accretion that leads to the galaxy formation can be separated into two different phases (e.g. Kereš et al. 2005; Dekel et al. 2009a,b; Khochfar \& Silk 2009; van de Voort et al. 2011; Faucher-Giguère et al. 2011). On the one hand, we distinguish a cold mode where the gas is accreted through the filamentary streams. The cold mode dominates the growth of galaxies at high redshifts, and the growth of lower mass objects at any times. On the other hand, in more massive haloes $\left(M_{\mathrm{h}}>10^{12} M_{\odot}\right)$ and at low $z$, the accretion is dominated by a hot mode, where a 
large fraction of the gas is shock-heated to temperatures close to the virial temperature. This gas feeds a hot stable atmosphere $\left(T_{\mathrm{g}}>10^{5} \mathrm{~K}\right)$ around the central host galaxy. To take into account this bimodal accretion, we use Lu et al. (2011) prescription (their Eqs. (24) and (25)). The accreted mass, divided into the two modes, is stored in two different reservoirs $M_{\text {cold }}$ and $M_{\text {hot }}$. The two reservoirs feed the galaxy with rates close to the free-fall rate for the cold mode and follows a cooling process for the hot mode.

\subsection{Disc formation}

Accretion from cold streams and cooling flows feed the galaxy disc in the centre of the dark-matter halo. We assume that this cold gas initially forms a thin exponential disc. Gas acquires angular momentum during the mass transfer (Peebles 1969). After its formation, the disc is supported by its angular momentum. This paradigm is based on the prescription given by Blumenthal et al. (1986) or Mo et al. (1998), and has been frequently used in SAMs, as in Cole (1991), Cole et al. (2000), Hatton et al. (2003), or Somerville et al. (2008).

Since more than one decade, observations (Cowie et al. 1995; van den Bergh 1996; Elmegreen \& Elmegreen 2005; Genzel et al. 2008; Bournaud et al. 2008) and hydrodynamic simulations (Bournaud et al. 2007; Ceverino et al. 2010, 2012) show the existence of gas-rich turbulent discs at high $z$. These discs are unstable and undergo gravitational fragmentation that forms giant clumps. These clumps interact and migrate to the centre of the galaxy where they form a pseudo-bulge component (Elmegreen 2009; Dekel et al. 2009b). In our model, we use a new self consistent model of disc instabilities. We assume that, in the disc, mass over-density and low-velocity dispersion lead to the formation and migration of giant clumps. A complete description of this process, which is based on Dekel et al. (2009b) and which has been adapted to our SAM approach, is given in a companion paper (Cousin et al. 2015). In brief, we compute the instantaneous unstable disc mass using the Toomre criterium (Toomre 1963, 1964). This unstable mass (mass in clumps) increases with time following the evolution of the disc. When this mass becomes higher than a mass threshold corresponding to a characteristic individual clump mass (Dekel et al. 2009b), we compute the transfer of the clump mass from the disc to the pseudo-bulge component. This transfer is modelled as a micromerger event with the pre-existing bulge component.

\subsection{Star formation}

In each galaxy component, disc and/or bulge, the cold gas mass $M_{\mathrm{g}^{\star}}$ is converted into stars. In standard models, the totality of the cold gas can be converted into stars. In Sect. 4.2, we present a strong modification of this prescription by introducing a nostar-forming gas component $\left(M_{\mathrm{g}}\right)$. In anticipation to this change, we specify here that obviously only the star-forming gas component $\left(M_{\mathrm{g}^{\star}}\right)$ takes part in the star formation process. We use the following standard definition of the SFR:

$\dot{M}_{\star}=\varepsilon_{\star} \frac{M_{\mathrm{g}^{\star}}}{t_{\mathrm{dyn}}}$

where $\varepsilon_{\star}(=0.02)$ is a free parameter adjusted to follow the Schmidt-Kennicutt relation (Kennicutt 1998), and $t_{\mathrm{dyn}}$ is the dynamical time given by

$t_{\text {dyn }}= \begin{cases}\operatorname{MIN}\left(2 r_{1 / 2} \sigma_{\mathrm{v}}^{-1}, 2 \pi r_{1 / 2} V_{\mathrm{c}}^{-1}\right): & \text { for disc } \\ 2 r_{1 / 2} \sigma_{\mathrm{v}}^{-1}: & \text { for bulge }\end{cases}$ where $V_{\mathrm{c}}$ is the circular velocity measured at the half radius mass, and $\sigma_{\mathrm{v}}$ is the mean velocity dispersion. For completeness, we add that the star formation is computed only if the projected star forming gas surface density $\Sigma_{\mathrm{g}}$ is higher than a given threshold $\log _{10}\left(\Sigma_{\mathrm{g}}^{\mathrm{min}}\right)=1\left[M_{\odot} \mathrm{pc}^{2}\right]$.

\subsection{Supernovae feedback}

In a given stellar population, massive stars evolve quickly and end their life as supernovae. This violent death injects gas and energy into the interstellar medium. The gas is heated, and a fraction can be ejected from the galaxy plane and feed the surrounding host-halo phase. In addition, these ejecta are at the origin of the metal enrichment of structures. Supernova feedback is therefore a crucial ingredient. In the majority of SAM (e.g. Kauffmann et al. 1993; Cole et al. 1994, 2000; Silk 2003; Hatton et al. 2003; Somerville et al. 2008), and according to some observational studies (e.g. Martin 1999; Heckman et al. 2000; Veilleux et al. 2005), the SN-reheating or SN-ejecta rate is linked to the SFR. As proposed by Dekel \& Silk (1986), we computed the ejected mass rate due to supernovae by using kinetic energy conservation. Another paradigm based on momentum conservation could be used, but it has been shown by Dutton \& van den Bosch (2009) that, in the low-mass regime, the energydriven feedback is more efficient and leads to better results.

The ejected mass rate $\dot{M}_{\mathrm{ej}, \mathrm{SN}}$ due to $\mathrm{SN}$ is linked to the SFR $\dot{M}_{\star}$ by using the individual supernova kinetic energy as:

$\dot{M}_{\mathrm{ej}, \mathrm{SN}} V_{\text {wind }}^{2}=2 \varepsilon_{\mathrm{ej}} \eta_{\mathrm{sn}} E_{\mathrm{sn}} \dot{M}_{\star}$

where we use $\eta_{\mathrm{sn}}=9.3 \times 10^{-3} M_{\odot}^{-1}$ and an efficiency $\varepsilon_{\mathrm{ej}}=0.3^{1}$. The value used for this parameter is similar to the one applied in standard SAMs (e.g. Somerville et al. 2008; Guo et al. 2011).

To break the degeneracy between the ejected mass and the velocity of the wind, we must add a constraint on the wind velocity. We rely on Bertone et al. (2005) in which the wind velocity is linked to the star formation rate (Martin 1999). It seems to be independent of the galaxy morphology (Heckman et al. 2000; Frye et al. 2002). We therefore use Eq. (9) in Bertone et al. (2005) to model the wind velocity.

On average, wind velocities obtained with this prescription are higher than in other studies (e.g. Somerville et al. 2008; Dutton \& van den Bosch 2009). Indeed it is common to use galaxy escape velocity to describe the wind, which is, for the ejection process, the minimum required value. Therefore, the ejected mass is at its maximum (see Dutton \& van den Bosch 2009, their discussion in Sect. 7.3). Consequently, our loading factor $\left(\dot{M}_{\mathrm{ej}, \mathrm{SN}} / \dot{M}_{\star}\right)$ is smaller than in other models, and therefore our mean ejected mass is also lower. The difference between our reference model and standard supernova feedback is discussed in Sect. 3.2.

\subsection{The active galaxy nucleus}

A supermassive black hole $(\mathrm{SMBH})$ can evolve in the centre of the bulge. We form the seed of the SMBH by converting a fraction of the bulge mass (gas and stars) to the SMBH mass,

1 The influence of the efficiency value has been tested in the range $\varepsilon_{\text {ej }} \in[0.05,10]$. Obviously a strong increase in the SN-efficiency increases the amount of ejected gas. The star formation activity is therefore reduced, but this effect affects only the amplitude and not the shape of the stellar mass function. Moreover, looking at the amplitude, its decrease is not enough to be in agreement with the observations. 
when the bulge mass becomes higher than a mass threshold $M_{\text {bulge }} \geq 10^{3} M_{\text {bh }} M_{\odot}$. This formation process is only turned on during a merger event. The SMBH formed at this time has a mass equal to $M_{\mathrm{bh}}=10^{3} M_{\odot}$. This mass is created by instantaneously converting a fraction of the gas and stars in their respective ratio. After its formation, the SMBH evolves in the centre of the bulge by accretion and clumps migration. The accretion process is mainly driven by the (Bondi 1952) accretion prescription, and we add an episodic accretion linked to clumps migration to this classical mechanism. This accretion is obviously limited by the maximum value of the Eddington accretion rate. As for supernovae, AGNs produce winds and contribute to the hot-atmosphere heating. We convert a given fraction of the power produced by mass accretion into kinetic and thermal power $\left(f_{\text {Kin }}=10^{-3}\right.$, e.g. Proga et al. 2000; Stoll et al. 2009; Ostriker et al. 2010). To compute the AGN ejected mass rate, we use the same kinetic conservation criterium as applied to supernovae: $\dot{M}_{\text {jet }} V_{\text {jet }}^{2} \propto \dot{M}_{\text {agn }} c^{2}$. Then we compute the momentum transfer between the AGN jet and the gas to estimate the mass that leaves the galaxy owing to AGN/gas coupling. We assume that all the mass is ejected with a velocity equal to the galaxy escape velocity $\left(\dot{M}_{\text {ej,AGN }} \propto \dot{M}_{\text {jet }} \frac{V_{\text {jet }}}{V_{\text {esc }}}\right)$. As explained in Sect. 2.7, the thermal power of the AGN is used for the monitoring of the hot phase temperature.

\subsection{Hot-halo phase}

As mentioned previously, galaxies hosted by massive dark matter haloes present a hot stable atmosphere generated by the hot cosmological accretion and maintained by galaxy ejecta. We use a new self consistent model for the hot halo phase evolution. We follow the hot halo phase mean temperature $(\bar{T})$ by applying a conservation criterion on the energy produced by hot accretion and/or feedback winds coming from SN/AGN. For SN and AGN, a fixed fraction $\left(f_{\text {Therm }}=5 \%\right.$ ) of the non-kinetic energy is converted into wind-thermal energy.

In parallel to the mean temperature monitoring, we compute the evaporated mass ${ }^{2}$ and mass loss due to galactic winds. All these calculations are done in a dominant dark-matter gravitational potential and assume the hydrostatic equilibrium (HEC; Suto et al. 1998; Makino et al. 1998; Komatsu \& Seljak 2001; Capelo et al. 2012). This new model gives a mean temperature $(\bar{T})$ close to the standard virial temperature prescription for structure in the intermediate dark matter halo mass range $\left(10^{10}-10^{12} M_{\odot}\right)$. Low-mass structures have higher temperatures $\left(\leq 2 T_{\mathrm{vir}}\right)$. This result is linked to the gas ionization fraction that is assumed to be the same for all structures. Massive structures that host an active galaxy nucleus also have higher mean temperatures, but never higher than three times the temperature derived from the standard virial assumption.

\subsection{Cooling processes}

Cooling is computed using the classical model initially proposed by White \& Frenk (1991). The condensed mass enclosed in the cooling radius $r_{\text {cool }}$ is estimated assuming:

- an HEC gas profile $\rho_{\mathrm{g}}(r)$ (Suto et al. 1998; Makino et al. 1998; Komatsu \& Seljak 2001; Capelo et al. 2012),

\footnotetext{
2 The hot atmosphere is considered in hydrostatic equilibrium in the dark matter halo potential well. We use a Maxwell-Boltzmann function to describe the velocity distribution. At each time step, the mass corresponding to the hot gas that has higher velocities than the escape velocity of the halo is definitively removed.
}

- a mean constant temperature $\bar{T}$,

- a temperature and metal dependent cooling function $\Lambda\left(T, Z_{\mathrm{g}}\right)$ (Sutherland \& Dopita 1993).

The cooling radius $r_{\text {cool }}$ is the unique solution for $t\left(r_{\text {cool }}\right)=t_{\text {cool }}$, where $t_{\text {cool }}$ is the effective cooling time computed as the life time of the hot gas phase, and $t(r)$ is the cooling time function of White \& Frenk (1991)

$$
t(r)=0.64 \frac{m_{\mathrm{p}} k_{\mathrm{b}} \bar{T}}{\rho_{\mathrm{g}}(r) \Lambda\left[\bar{T}, Z_{\mathrm{g}}\right]} .
$$

\subsection{Mergers and bulge growth}

At a given time step $t_{n}$, if two or more haloes have the same descendant at $t^{n+1}$, these haloes and their host galaxies have merged during this time lapse. We assume that the merger occurs at $t_{\text {merge }}=0.5 \times\left(t^{n+1}+t_{n}\right)^{3}$. Between $t_{n}$ and $t_{\text {merge }}$, the progenitors evolve in their host dark-matter halo and between $t_{\text {merge }}$ and $t^{n+1}$ the remnant galaxy evolves in the descendent dark-matter halo. Even if more than two progenitors are identified, mergers are computed using dark-matter (and associated galaxy) pairs starting from the lower sub-halo mass to the higher main halo mass. The post-merger galaxy morphology depends on the mass (galaxy + dark-matter halo) ratio of the two progenitors. We define

$\eta_{\text {merger }}=\frac{\operatorname{MIN}\left(M_{1 / 2,1} ; M_{1 / 2,2}\right)}{\operatorname{MAX}\left(M_{1 / 2,1} ; M_{1 / 2,2}\right)}$

where $M_{1 / 2, i}=M_{\mathrm{gal}, i}\left(r<r_{1 / 2}\right)+2 M_{\mathrm{dm}, i}\left(r<r_{1 / 2}\right)$ is, for system $i$, the sum of the galaxy and the dark matter halo mass enclosed in the galaxy half-mass radius $\left(r_{1 / 2}\right)$.

For $\eta_{\text {merge }}<0.25$, we consider that it is a minor merger. In this case, the disc and the pre-existing bulge component are kept, and gas and star contents are just added. The remnant disc size is set to the larger disc progenitor size. We apply the same rule to the bulge component. The velocities (dispersion and circular) of the bulge and the disc are recomputed with the properties of the remanent dark-matter halo. In the case of a major merger, $\eta_{\text {merge }}>0.25$, progenitor discs are destroyed, and the remanent galaxy is only made of a bulge. The half mass radius of this spheroid is computed using the energy conservation and the virial theorem (as in Hatton et al. 2003). Like a pseudo-bulge component formed by giant clumps migration (Sect. 2.3), bulges are described by a Hernquist (1990) model. After a major merger event, all new accreted material generates a new disc component. The mass is only transferred to the bulge by disc instabilities (Sect. 2.3).

Mergers are violent events. On a short time scale, the galaxy properties are strongly modified, and secular evolution laws (efficiencies) are no longer valid. To take the modifications induced by a merger into account, we use a boost factor, $\operatorname{MAX}\left[1, \varepsilon_{\text {boost }}(\Delta t)\right]$, that increases the efficiencies of star formation in each component of the galaxy (disc and bulge), and of $\mathrm{SMBH}$ accretion. The boost factor is defined as:

$\varepsilon_{\text {boost }}(\tau)=100 \eta_{\text {merger }} \eta_{\text {gas }} \exp \left(-\frac{\tau}{\tau_{\text {merger }}}\right)$

\footnotetext{
3 However, even if we accurately follow the sub-haloes and if the merging time laps is reduced, the instantaneous merging of galaxies is always a strong assumption and could be explored in detail in a future work.
} 
Table 1. List of SAMs compared in this paper.

\begin{tabular}{lll}
\hline \hline Model & Definitions/Comments & Colour plots \\
\hline$m_{0}$ & Okamoto et al. (2008), without (sn/agn)-feedback & red \\
$m_{1}$ & Okamoto et al. (2008) photoionization and our (sn/agn)-feedback processes (reference) & orange \\
$m_{2}$ & Gnedin (2000) photoionization and our (sn/agn)-feedback processes & green \\
$m_{3}$ & Gnedin (2000) photoionization and Somerville et al. (2008) SN-feedback, without AGN-feedback & cyan \\
$m_{4}$ & reference + no-star-forming gas disc component (Sect. 4.2) & purple \\
\hline
\end{tabular}

Table 2. List of the main models parameters. The values given here are identical or very similar to those commonly used in the literature.

\begin{tabular}{lll}
\hline \hline Symbol & Definition & Value \\
\hline$\varepsilon_{\star}$ & Star formation efficiency (Eq. (1)) & 0.02 \\
$\varepsilon_{\text {ej }}$ & SN feedback efficiency (Eq. (3)) & 0.3 \\
$\tau_{\text {merger }}$ & Merger time scale (Eq. (6)) & 0.05 Gyr \\
$\left\langle f_{\mathrm{b}}\right\rangle$ & Universal baryonic fraction (Eq. (7)) & 0.18 \\
\hline
\end{tabular}

where $\eta_{\text {gas }}$ is the gas fraction in the post-merger structure, $\tau$ is the time elapsed since the last merger event, $\tau_{\text {merger }}=0.05 \mathrm{Gyr}$ is a characteristic merger-time scale and $\eta_{\text {merger }}$ is given in Eq. (5). The formulation is used to simulate a time-dependent gas compression (decreasing with time) and takes the gas content of the two progenitors into account. The more gas they contain, the more the gas compression is high.

\subsection{The adaptive time-step scheme}

In a galaxy, various processes act at the same time on various time scales. For example, it is not efficient to compute the evolution of the cold filamentary phase, which evolves on a typical dark-matter dynamical time $\left(10^{6} \mathrm{yr}\right)$, with a time step following the ejection rate of the galaxy $\left(10^{4} \mathrm{yr}\right)$. Using the same time step for all components generates numerical errors on the slowly evolving component and degrades the precision. Each component of the baryonic halo or of the galaxy (disc and/or bulge) must evolve with a time step that is as close as possible to its dynamical time. Accordingly, we have developed an adaptive time-step scheme. Each halo or galaxy component has a separated evolution scheme, and interacts with others only if the mass transfers significantly affect its evolution. We consider that the mass reservoir is modified if the variation is over $10 \%$.

\section{Star formation in the low-mass structures in standard models}

We focus in this section on the main problem of star formation activity in low-mass structures. We explore various prescriptions for the photoionization or SN-feedback processes and compare the results with some fundamental galaxy properties: stellar mass function (SMF), $M_{\mathrm{h}}$ versus $M_{\star}$ relation (SHMR), the sSFR versus $M_{\star}$ relation, and SFR distribution (or IR-LF).

Table 1 gives the description of the models, from $m_{0}$ without feedback processes (in red) to $m_{4}$, where we put a large fraction of the gas into a no-star-forming gas component (see Sect. 4.2). In the low-mass range analysed here, AGN-feedback does not play an important role, and therefore it is not further discussed.

\subsection{Impact of photoionization}

Gas heating generated by the first generation of stars and quasars limits the baryonic gas accretion in the smaller structures
(Kauffmann et al. 1993). The effective baryonic fraction $f_{\mathrm{b}}^{\mathrm{ph}-\text { ion }}$ (Eq. (7)) depends on both the redshift and the dark matter halo mass. The most commonly used formulation is the one proposed by Gnedin (2000) and Kravtsov et al. (2004):

$$
f_{\mathrm{b}}^{\mathrm{ph}-\text { ion }}\left(M_{\mathrm{h}}, z\right)=\left\langle f_{\mathrm{b}}\right\rangle\left[1+\left(2^{\alpha / 3}-1\right)\left(\frac{M_{\mathrm{h}}}{M_{\mathrm{c}}(z)}\right)^{-\alpha}\right]^{-3 / \alpha} .
$$

In this definition, $\left\langle f_{\mathrm{b}}\right\rangle$ is the universal baryonic fraction, $M_{\mathrm{h}}$ the dark matter halo mass, and $M_{\mathrm{c}}(z)$ the filtering mass corresponding to the mass where the halo lost half of its baryons. Finally, $\alpha$ is a free parameter that mainly controls the slope of the transition.

$$
\begin{aligned}
& \text { - For } \alpha=1, f_{\mathrm{b}}^{\mathrm{ph}-\text { ion }} /\left\langle f_{\mathrm{b}}\right\rangle: 0 \rightarrow 1 \text { for } M_{\mathrm{h}}: 10^{9} \rightarrow 10^{12} M_{\odot}, \\
& \text { - For } \alpha=2, f_{\mathrm{b}}^{\text {ph-ion }} /\left\langle f_{\mathrm{b}}\right\rangle: 0 \rightarrow 1 \text { for } M_{\mathrm{h}}: 10^{9} \rightarrow 10^{10} M_{\odot} .
\end{aligned}
$$

The redshift evolution of the filtering mass $M_{\mathrm{c}}$ and the value of $\alpha$ are the crucial parameters governing the impact of photoionization on small structures.

These parameters have been constrained using hydrodynamic simulations that include UV photoionization performed by, for example, Gnedin (2000), Kravtsov et al. (2004), Hoeft et al. (2006), or Okamoto et al. (2008). The analysis of these different simulations gives different results, and therefore various parameter values and filtering mass behaviours. While in Gnedin (2000) the slope index is set to $(\alpha=1)$, Okamoto et al. (2008) find a higher value $(\alpha=2)$. We recall in Appendix B the mathematical expressions for the two filtering masses given in these papers and used here.

In Fig. 1, we show the evolution of $f_{\mathrm{b}}^{\mathrm{ph}-\text { ion }} /\left\langle f_{\mathrm{b}}\right\rangle$ for the two prescriptions. The two top panels are dedicated to the Gnedin (2000) model. Their filtering mass definition is the one most commonly used in the literature (Somerville 2002; Somerville et al. 2008, 2012; Croton et al. 2006; Guo et al. 2011). In the upper panel, we apply $z_{\text {reion }}=10$, as in Somerville et al. (2012). In the central panel we apply $z_{\text {reion }}=9$ to compare with Okamoto et al. (2008). The grey horizontal line marks a decrease of $50 \%$ comparing to the universal baryonic fraction. We consider that the photoionization effect is important when $f_{\mathrm{b}}^{\mathrm{ph}-\mathrm{ion}}<0.5\left\langle f_{\mathrm{b}}\right\rangle$. The colour code indicates the redshift evolution.

At our dark matter halo mass resolution, in the first case (Gnedin 2000, $\left.z_{\text {reion }}=10\right)$, photoionization starts to play a role at $z \simeq 8$. At low $z$, the small structures are strongly affected by the photoionization process. This trend is still true when the reionization redshift is decreased (central panel, $z_{\text {reion }}=9$ ). The effect of photoionization is much less important in the Okamoto et al. (2008) prescription. Indeed, the significant decrease in $f_{\mathrm{b}}^{\mathrm{ph}-\text { ion }} /\left\langle f_{\mathrm{b}}\right\rangle$ only appears at the mass resolution for redshift $z<1$. In this case, the gas heating due to the UV background cannot affect, at high redshift, the baryonic assembly of small structures. This difference in behaviour comes from the different redshift evolutions of the filtering mass $M_{\mathrm{c}}(z)$. In the two cases, the authors use hydrodynamic simulations to constrain this evolution. 


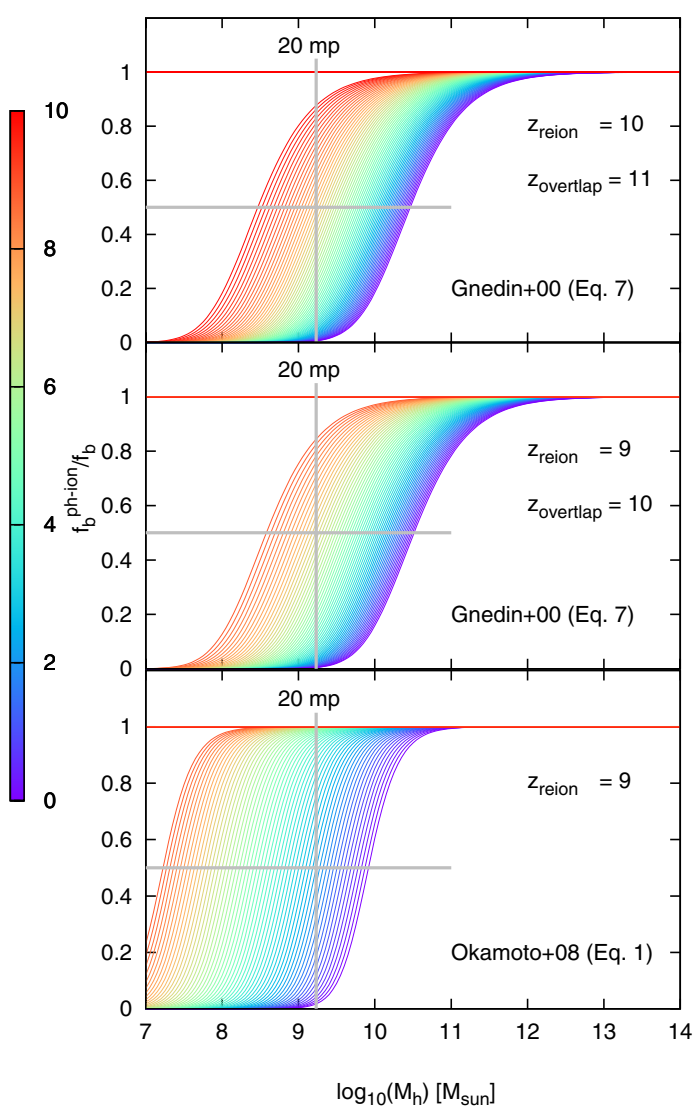

Fig. 1. Normalized baryonic fraction $f_{\mathrm{b}}^{\mathrm{ph}-\text { ion }} / f_{\mathrm{b}}$ as a function of the dark matter halo mass. The colour code shows the redshift evolution (from $z=10$ in red, to $z=0$ in purple). We compare two different prescriptions for the evolution of the baryonic fraction after the redshift of reionization. The two upper panels are made using the Gnedin (2000) prescription with two different reionization redshifts (zreion $=10$ and 9). The bottom panel shows the Okamoto et al. (2008) prescription with zreion $=9$. The grey vertical line indicates our dark-matter mass resolution limits (20 dm-particles). In the Gnedin (2000) model (central panel) the strong effects induced by photoionization $\left(f_{\mathrm{b}}^{\mathrm{ph}-\text { ion }}<0.5\left\langle f_{\mathrm{b}}\right\rangle\right)$ appear at $z \simeq 7-8$ and become stronger with decreasing redshift. In the Okamoto et al. (2008) prescription, the impact of photoionization comes much later $(z<1)$ and cannot affect the galaxy formation processes at high $z$.

As explained by Okamoto et al. (2008), even if the two studies use different assumptions (e.g. link between gas density and gas temperature), it seems that the difference between the two prescriptions is most likely due to insufficient resolution of the Gnedin (2000) simulation.

We applyed the two photoionization prescriptions in models $m_{1}$ and $m_{2}$. The first one, $m_{1}$, uses the Okamoto et al. (2008) description. It is our reference model. For comparison, we use Gnedin (2000) prescription in $m_{2}$. As we can see in Figs. 3 and 4, the two models mainly have an impact at low redshift. The figures show that the Gnedin (2000) prescription reduces the stellar mass formed in the small dark matter mass regime more than does the Okamoto et al. (2008) prescription. This is consistent with the baryonic fraction behaviour described above. Gas accretion is more reduced in the Gnedin (2000) model. At low halo mass $\left(M_{\text {halo }}<5 \times 10^{10} M_{\odot}\right)$ and at low redshift $(z<2)$, the mean stellar mass built through the Gnedin (2000) photoionization model $\left(m_{2}\right)$ may be ten times lower than the one built with the Okamoto et al. (2008) model $\left(m_{1}\right)$.
Currently, the majority of SAMs use the Gnedin (2000) photoionization parameterization. The parameter set $\left(\alpha, M_{\mathrm{c}}(z)\right)$ used in this case leads to an accretion rate on the galaxies that is reduced compared to what is obtained using Okamoto et al. (2008). This result fully agrees with Guo et al. (2011). However, regardless of the case, it is evident from Fig. 3 that photoionization is not enough to reduce the low-mass end of the stellar mass function as required by the observations.

\subsection{Impact of SN feedback}

While the photoionization process reduces the gas feeding of the galaxy, supernova feedback expels the gas already present in the galaxy. Despite their different actions, both processes tend to reduce the amount of gas available to form stars.

We compare two SN-feedback models:

- our model based on kinetic, thermal energy conservation, hot gas phase heating, and evaporation;

- the Somerville et al. (2008) model based on their Eqs. (12) and (13) (reheated rate and escape fraction). In this case, the hot gas-phase temperature is not monitored as it is in our model, but is set to the dark matter virial temperature.

As listed in Table $1, m_{2}$ and $m_{3}$ used the same photoionization prescription (Gnedin 2000). They differ only in their SNfeedback model. A simple comparison between the stellar mass functions (Fig. 3) given by $m_{2}$ and model $m_{3}$ in which we have implemented the Somerville et al. (2008) prescription indicates that the $\mathrm{SN}$-feedback mechanism is more efficient in their model. This difference is even more visible on the SHMR (Fig. 4) which indicates that the stellar mass produced in low-mass haloes $\left(M_{\mathrm{h}}<10^{11} M_{\odot}\right)$ is, on average, higher in our model by a factor close to 3 . This difference decreases when $z$ decreases and $M_{\mathrm{h}}$ increases.

As explained previously (Sect. 2.5), the higher mean wind velocity computed in our model following Bertone et al. (2005) leads to a lower ejected mass for a given kinetic energy. A more detailed comparison of the two models proves that the reheating rate computed with the Somerville et al. (2008) SN-feedback model $\left(m_{3}\right)$ is, on average, for a given dark matter halo mass, twice more than with our ejected-rate $\left(m_{2}\right)$. We see the same trend if we compare the two models at a fixed SFR.

The ejected-hot gas is transferred to the hot-halo phase. Its possible definitive ejection from the hot atmosphere is computed by assuming the dark-matter potential well, taking the velocity of the wind and the escape velocity of the dark matter structure into account. The gas mass that is definitively ejected is higher in $m_{2}$ than in $m_{3}$, on average, in the intermediate range of masses. This is linked to the wind velocity that is fixed to a value of about $\simeq 100-150 \mathrm{~km} \mathrm{~s}^{-1}$ in $m_{2}$ (see Eq. (13) in Somerville et al. 2008) and more than $\sim 150 \mathrm{~km} \mathrm{~s}^{-1}$, on average, in $m_{3}{ }^{4}$. This difference is at the origin of the break in the slope of the stellar-mass function between $m_{2}$ and $m_{3}$.

The large difference, at high mass, between $m_{2}$ and $m_{3}$ is due to the AGN feedback. For consistency reasons with our hot gas phase heating modelling (which associates both SN and AGN), we cannot apply the AGN-feedback processes in this Somerville et al. (2008) model comparison, while, in model $m_{2}$, our AGN feedback is turned on, and therefore reduces the stellar mass.

Despite the different parameterizations and energy injection scales for supernovae, currently the classical semi-analytical

\footnotetext{
4 For a given kinetic energy the larger the wind velocity, the smaller the mass ejected mass.
} 
models do not seem to be able to explain the high-redshift behaviour of the mass function in the low-mass range (see also Fig. 23 in Guo et al. 2011; and Fig. 11 in Ilbert et al. 2013). Even if some SAMs, such as Somerville et al. (2008), Guo et al. (2011), or Henriques et al. (2013), use a dedicated parametrization to reproduce the galaxy properties at $z=0$, it seems that, at high redshift, the low-mass range problem of the stellar-mass function is not only linked to a SN-feedback efficiency calibration. Indeed, Guo et al. (2011; their Figs. 8 and 23) show that the number of low-mass star-forming galaxies are still larger than observed. A new ad-hoc parametrization of the Guo et al. (2011) model is proposed by Henriques et al. (2013). Using a very high efficiency for the SN feedback coupled to a very low efficiency for the re-accretion of the gas (see Sect. 4.3 for a complete discussion), they obtain a better result in the low-mass regime.

A strong increase in the $\mathrm{SN}$-wind efficiency in lowmass structures also leads to very high mass-loading factors $\left(\dot{M}_{\text {ej }} / \dot{M}_{\star}>10\right.$, Henriques et al. 2013, their Fig. 3). Such factors are much greater that those derived from spectroscopic observations (e.g. Sturm et al. 2011; Rubin et al. 2011; Bouché et al. 2012) even if the measurement of this parameter is difficult and is currently performed on massive systems. In these conditions, stellar outflows alone cannot limit the star formation sufficiently. In this context, the measurement of the mass-loading factor becomes a key point.

\section{An ad hoc recipe for reconciling models and observations}

At high redshift $(z>1)$, as shown in Fig. 3, the amplitude of the faint end of the stellar mass function is dramatically overestimated by the models $\left(m_{1}, m_{2}\right.$ and $\left.m_{3}\right)$. This result is consistent with the overestimate of stellar mass in low-mass dark matter haloes: small structures form too many stars. In general, this problem is addressed by a strong SN feedback and/or photoionization. As shown previously, photoionization and SN-feedback cannot be sufficient to reduce significantly the star formation in low-mass objects. Strong feedback models give some good integrated results (at $z \simeq 0$ ) (Guo et al. 2011; De Lucia \& Blaizot 2007) but fail at higher redshift (see Ilbert et al. 2013, their Fig. 11).

In this section we propose a strong modification of implementing of the star-formation mechanism in our semi-analytical model to try to reconcile models and observations.

\subsection{Can all the cold gas form stars?}

In a standard semi-analytical model, the SFR is adjusted to follow the observed empirical Kennicutt (1998) law. The rate is computed following Eq. (1) and is applied to the entire cold gas reservoir. In this context, the efficiency parameter $\varepsilon_{\star}$ determines the fraction of star-forming gas. This fraction is obviously constant. The Kennicutt (1998) law reflects, with global variables, an overall view of the star formation process. Even if large reservoirs of gas are observed in galaxies, at least up to $z \simeq 1.5$ (e.g. Daddi et al. 2010), these observations do not give any information about the real fraction of the gas that is available to form stars.

The Kennicutt law and the homogeneous description of the cold gas cannot describe the complex structure of the ISM. Observations indicate that only a very small amount of the gas mass is used at a given time to form stars in galaxies (including ours); the star formation occurs only in highly-concentrated regions and not in the entire disc. Recent Herschel observations show that stars form in dense cold cores with a typical size of $0.1 \mathrm{pc}$, embedded in the interstellar filamentary structure (André et al. 2010; Heiderman et al. 2010; Lada et al. 2012). These prestellar cores are formed only when the gas surface density is higher than $\Sigma_{\mathrm{thr}} \simeq 160 M_{\odot} \mathrm{yr}^{-1}$. Observations show that only a small amount of the total gas mass $(\simeq 15 \%)$ is above this column density threshold and only a small fraction $(\simeq 15 \%)$ of this dense gas is in the form of prestellar cores (André 2013; André et al. 2014). Therefore, a large amount of the gas is not available to form stars. This no-star-forming component corresponds to the gas that is occupying the low levels of matter structuration, where the gas surface density is low.

\subsection{The no-star-forming disc component}

When accreted on the galaxy disc, the surface density of fresh gas (considered as homogeneously distributed) is low. Progressively the gas, controlled by the turbulence and gravity energy balance, is structured more and more (Kritsuk \& Norman 2011). The energy injected by the accretion process must be dissipated before star-formation process can start. Since the dissipation scale is much smaller than the energy injection scale, we assume that the energy cascade introduces a delay between the accretion time and the star formation time.

To model this process, we introduce a model $m_{4}$ with a new gas component in galaxy discs: the no-star-forming gas. The delay between the accretion time of fresh gas and the time when this gas is converted into stars is modelled by a transfer rate between the no-star-forming gas and the star-forming gas reservoir $\left(\mathrm{g}^{\star}\right)$ that follows

$\dot{M}_{\mathrm{g}^{\star}, \text { in }}=\dot{M}_{\mathrm{g}, \text { out }}=\varepsilon_{\star} \min \left[1,\left(\frac{M_{\mathrm{h}}}{10^{12} M_{\odot}}\right)^{3}\right] \frac{M_{\mathrm{g}}}{t_{\mathrm{dyn}}}$

where $M_{\mathrm{g}}$ is the mass of no-star-forming gas, $t_{\text {dyn }}$ the disc dynamical time, and $\varepsilon_{\star}$ an efficiency parameter, identical to the star formation efficiency (Eq. (1)). Obviously this formulation is totally ad hoc and does not provide any physical information on the link between the characteristic time of the turbulent cascade and the mass of the halo. The halo mass dependence in Eq. (8) is introduced to reproduce the shape of the stellar to halo mass relation, as observed in for example Leauthaud et al. 2012; Moster et al. 2010; Behroozi et al. 2010; Béthermin et al. 2012.This formulation has no other purpose. It does not describe the structuration of the density. However, the dependence in $M_{\mathrm{h}}^{3}$ indicates that the star formation regulation process must be extremely strong in the smallest structures. In the context of the bimodal accretion, the accretion is dominated by the cold mode below $M_{\mathrm{h}}=10^{12} M_{\odot}$. This cold accretion is feeding the no-starforming gas reservoir, which thus regulates the star formation in such structures.

In the context of this new prescription we decided to apply the merger boost factor (Eq. (6)) to the no-star-forming transfer process (Eq. (8)) and to the star formation. Indeed we consider that mergers increase the mean gas concentration instantaneously, and thus accelerate the structuration of the density.

If the no-star-forming gas was homogeneously added to the disc structure, the decrease in the star-forming gas fraction would be equivalent to a simple decrease in the star formation efficiency $\varepsilon_{\star}$. This is not satisfactory, and to maintain the star formation efficiency even with a large amount of no-star-forming gas, we thus had to adopt an artificial gas concentration, as illustrated in Fig. 2. Using this gas redistribution, we derive a new 

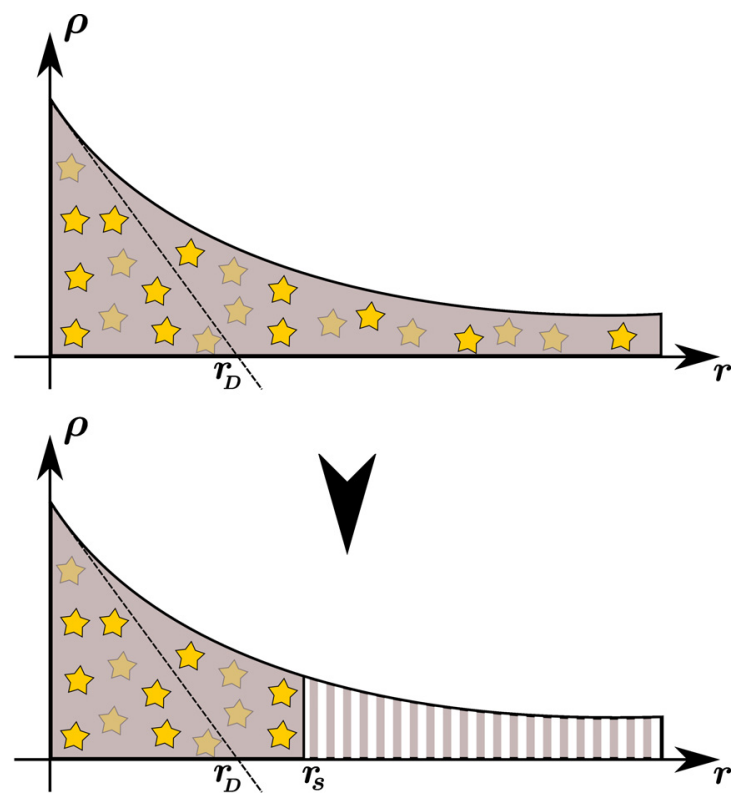

Fig. 2. Radial density profile of the gas in the disc. While in a classical model (upper panel) all the gas is available to form stars and is distributed in the whole disc, the star-forming gas is artificially concentrated in the centre of the disc and the no-star-forming is distributed in the outer region with our ad hoc model (lower panel). The star-forming gas is enclosed in the radius $r_{\mathrm{s}}$.

dynamical time, and thus SFR, from the circular velocity computed at the characteristic radius $r_{\mathrm{s}}$. With this model, we can produce high SFR, even if a large amount of gas is considered as no-star-forming, without modifying the star formation recipes.

\subsection{Comparison with the Munich model}

The current baseline of the Munich model is mainly described in Guo et al. (2011). Some important modifications are presented in Henriques et al. (2013). This paper focusses on the reincorporation of the ejected gas. The model is based on an ejecta reservoir that receives the gas ejected from the galaxy. The main hypothesis is that this gas is not available for cooling and it has to be reincorporated into the hot gas reservoir to cool. This ejecta reservoir, linked to the halo, is fed by the very efficient $\mathrm{SN}$-feedback processes. As presented in Henriques et al. (2013) the key point of this model is the reincorporation timescale. In the current model, it is inversely proportional to the halo mass and is independent of redshift (from $1.8 \times 10^{10} \mathrm{yr}$ for haloes with $M_{\mathrm{h}}=10^{10} M_{\odot}$ to $1.8 \times 10^{8} \mathrm{yr}$ for haloes with $\left.M_{\mathrm{h}}=10^{12} M_{\odot}\right)$. In this context, the gas expelled from lowmass structures is stored for a long time in the ejecta reservoir, so this model strongly limits the star formation process. In this scenario the no-star-forming gas is stored outside of the galaxy. This model gives good predictions in the low-mass range of the stellar mass function, but, without a prompt reincorporation of this gas in the cooling loop, the amount of star-forming gas, and therefore the star formation activity, are limited in the disc of low and intermediate-mass objects.

Regardless the mechanism behind it, it seems that the storage of the gas in a no-star-forming reservoir (e.g. reservoir of low-density gas in the disc, or reservoir without any cooling outside the galaxy) is the best way to modulate the star-formation efficiency such that semi-analytical models can reproduce the observations.

\section{Stellar and gas-mass assembly}

We have presented different processes that act on galaxy formation and, more precisely, on the star formation activity. We tested two photoionization models and two supernovae feedback models. In addition to these four models, we have proposed another model in which we have strongly limited the star formation efficiency in low-mass haloes. In this section we discuss the comparison of this model with the main galaxy properties, and compare the predictions of this model in detail with the other four.

\subsection{Stellar-mass function and SHMR}

We show in Fig. 3 the stellar-mass functions predicted by our models. Model outputs are compared with observational data from Ilbert et al. (2010, 2013), Baldry et al. (2008), and Yang et al. (2009), Caputi et al. (2011).

As discussed before, models $m_{1}, m_{2}$, and $m_{3}$ fail to reproduce the low-mass end of the stellar mass function. The disagreement is both on the amplitude (one order of magnitude higher at low mass) and on the shape of the mass function. Figure 4 shows the stellar mass as a function of the dark-matter halo mass. We compare all models with relations extracted from the literature (Behroozi et al. 2010; Béthermin et al. 2012; Moster et al. 2010). This figure indicates that the excess of galaxies with low stellar masses is due to an over-production of stars in the low-mass dark matter haloes. To reduce this tension, we applied a strong modification of the star formation process in $m_{4}$. The gas is kept in the disc but, a large amount of this gas cannot form stars. With this ad hoc model, in the low mass range, the levels of the stellar mass functions are in good agreement with observations for a wide range of stellar masses. This indicates that only a strong modification (a decrease in our case) of the mass of gas instantaneously available to form stars allows the star formation activity in low mass structures to be modulated and SAM to be reconciled with the observations.

Concerning the high-mass end of the stellar-mass function, all models under-predict the number of massive galaxies. For $z=4$ and $z=3$, the comparison with Ilbert et al. (2010) and Ilbert et al. (2013) observational mass functions indicate that the massive galaxies in our models are two time less massive than the observed distribution. This is also observed in other recent SAMs (see e.g. Henriques et al. 2013, their Figs. 4-6, and Guo et al. 2011, their Fig. 23). The only way to reconcile models and observation in this high-mass regime is to consider a model without any regulation mechanism (model $m_{0}$ ). In contrast, for the low-redshift range $(z=0-2)$, our models give a small excess for massive galaxies. This disagreement could be linked to an AGN-feedback that is not efficient enough. The volume in the simulated box $\left[(100 / h)^{3} \simeq 150^{3} \mathrm{Mpc}^{3}\right]$ does not allow us to have more than ten haloes with mass higher than $M_{\mathrm{h}}=10^{13} M_{\odot}$, and therefore there is a small statistical sample associated to this range of mass. As we can see in Fig. 3 at $z=0.3$ and for mass larger than $10^{11} M_{\odot}$, the stellar mass function is quite noisy. For information, we indicate, in each panel, the stellar mass above which uncertainties due to cosmic variance become larger than the differences between the models (see Appendix A for more details).

\subsection{Gas-mass function}

In $m_{4}$, we have chosen to modify the standard star formation paradigm, through the introduction of a delay between gas accretion and star formation. The step during which the 
M. Cousin et al.: Galaxy stellar mass assembly

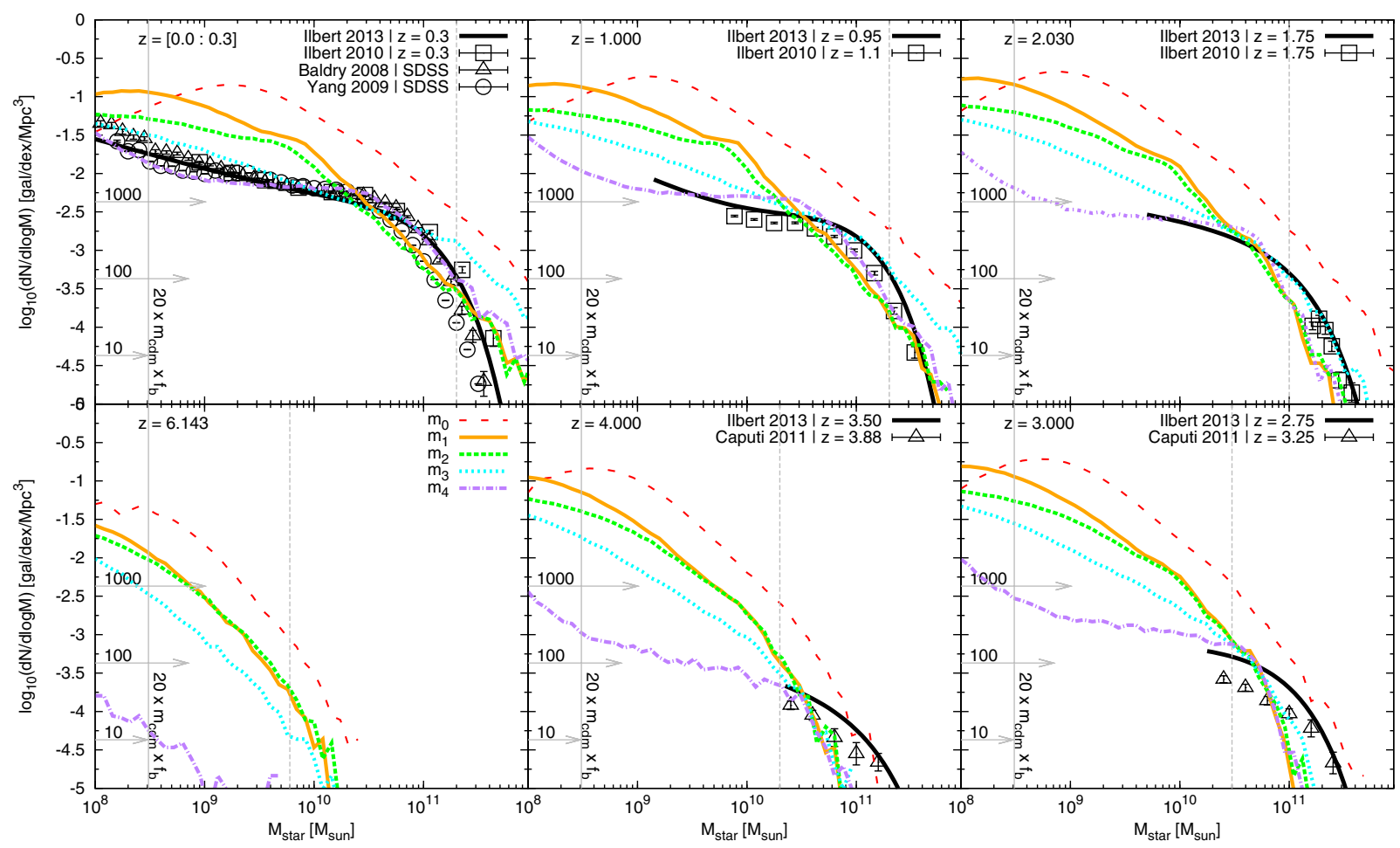

Fig. 3. Stellar mass function and its evolution with redshift. The redshift is increasing clockwise. The colour code is the same for all figures, and is detailed in the model list (Table 1). We compare our results with Ilbert et al. (2010, 2013; squares), Yang et al. (2009; circles), Baldry et al. (2008; triangles in the first panel), and Caputi et al. (2011; triangles) observations. Horizontal arrows show the link between the density and the number of haloes in our simulation volume. The grey dashed lines plotted in the high-mass range indicate the limit where uncertainties due to the cosmic variance are equal to the differences between models.

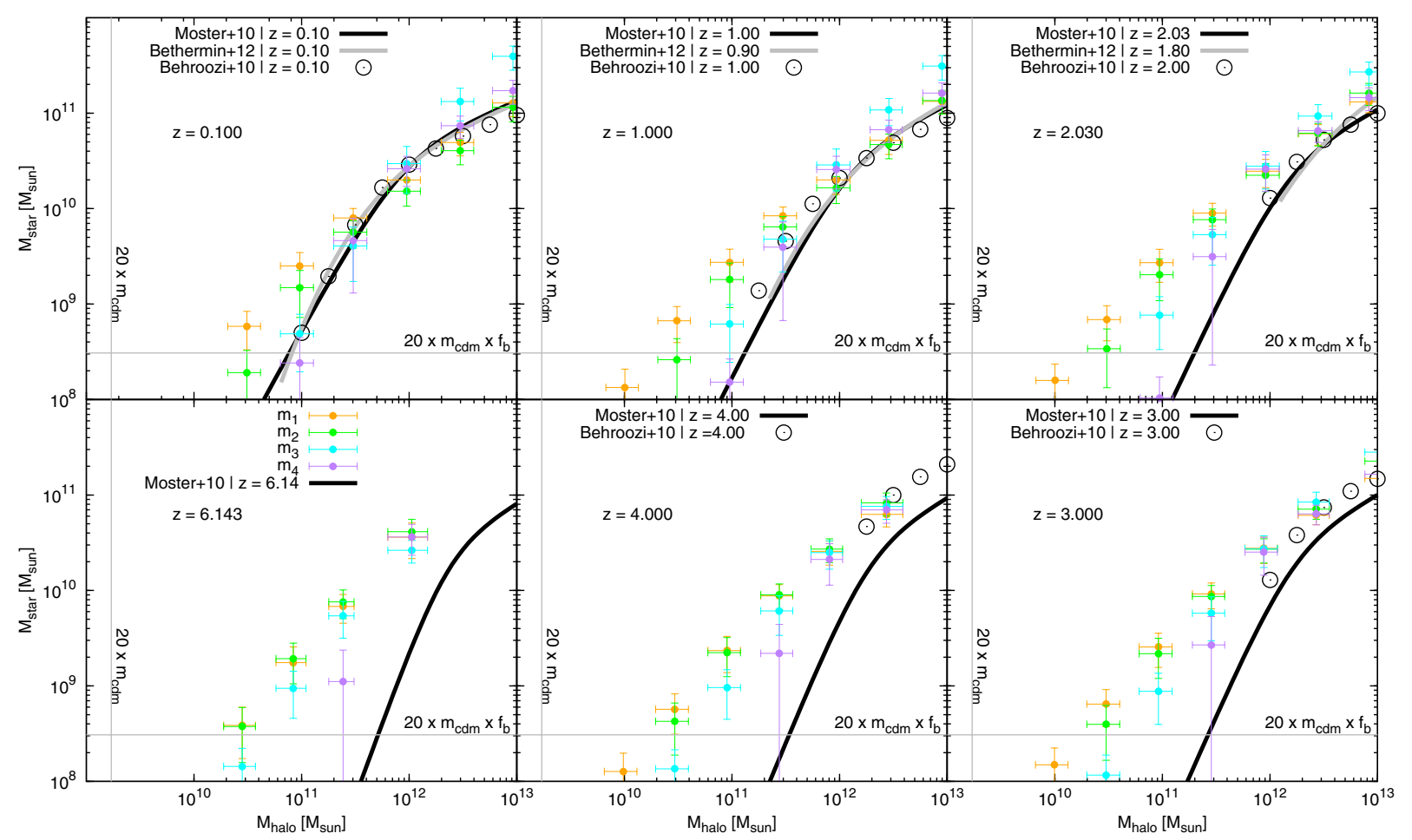

Fig. 4. Stellar-to-dark-matter halo mass relation (SHMR) for various redshifts. The models (coloured lines) are compared with recent analysis based on halo occupation or abundance matching (black line: Moster et al. 2010; grey line: Béthermin et al. 2012; black open circles: Behroozi et al. 2010). While standard models have a regular evolution of the stellar mass with $M_{\mathrm{h}}$, the ad hoc recipe included in $m 4$ produces: i) a very slow increase of the stellar mass for haloes with $M_{\mathrm{h}}<10^{11} M_{\odot}$; ii) a very strong increase of the stellar mass at intermediate halo mass $\left(10^{11}<\right.$ $M_{\mathrm{h}}<10^{12} M_{\odot}$ ). This shape is not captured well by our points with error bars. The large error bar associated with the $M_{\mathrm{h}}=10^{11} M_{\odot}$ point results from the very large scatter produced by the strong increase of the stellar mass in this halo mass range. iii) A slow increase in the stellar mass for $M_{\mathrm{h}}>10^{12} M_{\odot}$. 


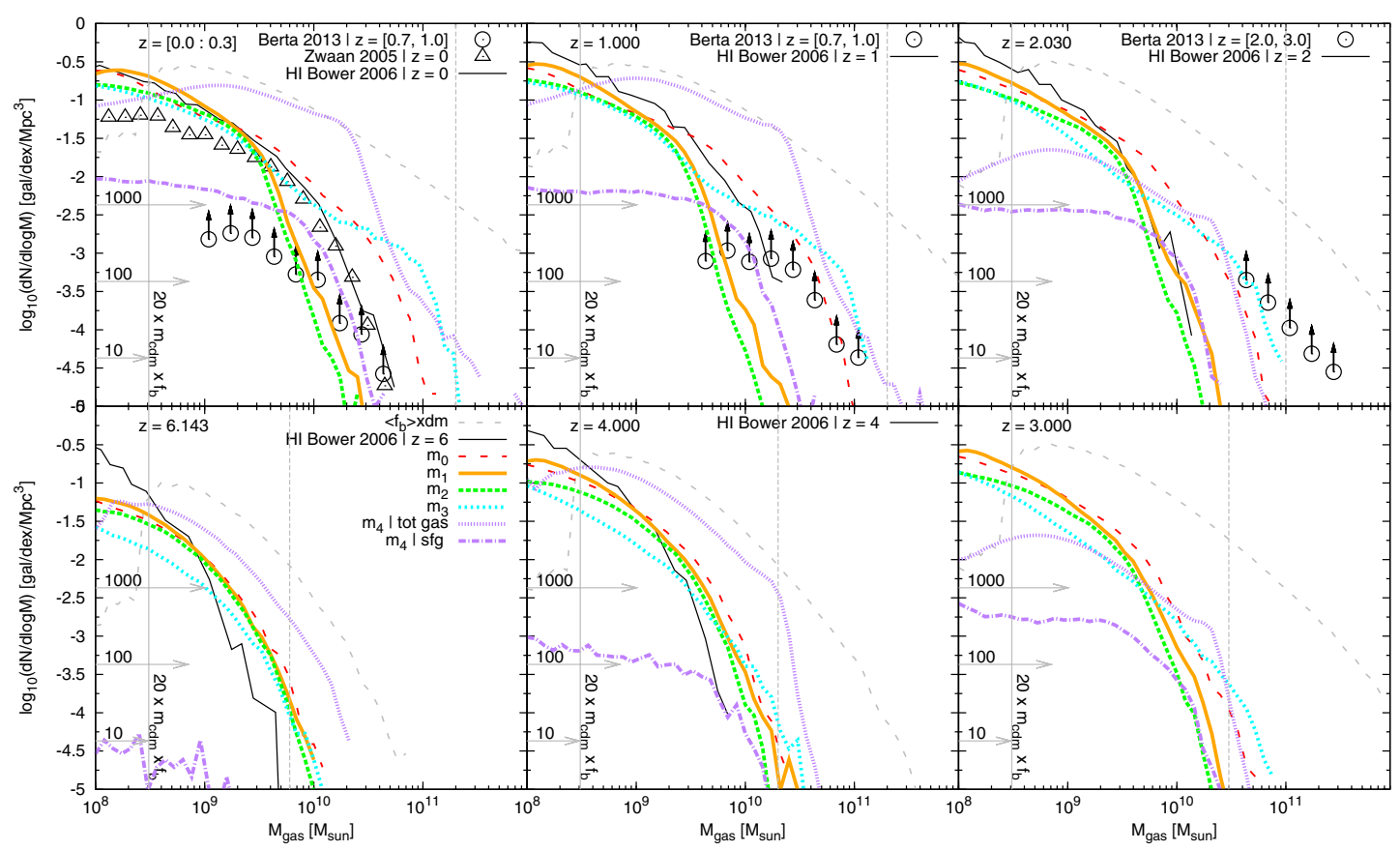

Fig. 5. Gas mass functions predicted by our SAMs. The colour code is explained in Table 1. For comparison, we add the extremal gas mass function deduced from the dark-matter mass function and the universal baryonic fraction $\left\langle f_{\mathrm{b}}\right\rangle$ (grey dashed-line). In the case of $m_{4}$, we plot the total (starforming + no-star-forming) and the star-forming gas mass function. We compare our results with the molecular gas mass function computed by Berta et al. (2013; lower limits, circles) and with the local HI mass function computed by Zwaan et al. (2005; triangles). The black solid line shows the HI mass function predicted by Lagos et al. (2011), using Bower et al. (2006) SAM. The horizontal arrows show the link between the density and the number of haloes in our simulation volume.

no-star-forming gas is converted into star-forming gas strongly reduces the star formation activity, and therefore the stellar mass build-up. Obviously the total amount of gas in galaxies (no-starforming and star-forming) will be strongly modified with respect to standard models. In this section, we compare the gas mass function predicted by all models with the available observational constraints.

In Fig. 5, we show the predicted gas-mass functions, together with the local HI mass function computed by Zwaan et al. (2005) and the molecular gas mass function coming from Berta et al. (2013). In their study, Berta et al. (2013) focus on the molecular gas contained in normal star-forming galaxies (within $\pm 0.5 \mathrm{dex}$ in SFR from the main sequence). Quiescent galaxies are therefore not taken into account. In these conditions, and as explained by Berta et al. (2013), their data points should be considered as lower limits. The gas-mass functions extracted from our models are computed using all galaxies contained in our simulated volume, and taking the total gas mass in galaxy discs into account (both star-forming and no-star-forming).

The gas-mass functions predicted by our reference model $m_{1}$ and its variation $\left(m_{2}\right)$ are very close. Indeed, the two models use the same prescription for gas ejection $(s n+a g n)$. At low mass and at all redshifts, the gas mass predicted by $m_{3}$ (using Somerville et al. 2008 sn feedback prescription) is also very close to $m_{1}$ and $m_{2}$. At high mass, the difference is due to $\mathrm{SMBH}$. Indeed, in $m_{3}$ the SMBH activity is not taken into account. Consequently, the cooling rate associated with high-mass haloes is not limited, and the amount of gas increases.

In the case of the new model $m_{4}$, we plot in Fig. 5 the total and the star-forming gas-mass function. As expected, the amount of total gas in $m_{4}$ is larger than in the reference model $m_{1}$ or its variations $\left(m_{2}\right.$ and $\left.m_{3}\right)$. The decrease in the star formation activity in $m_{4}$ leads to an large storage of the gas. In the first two panels $(z \simeq 0$ and $z \simeq 1)$, the difference between the total and the star-forming gas mass functions is larger than at higher redshift; the fraction of no-star-forming gas increases with time. This evolution is linked to the transfer rate between the no-starforming gas and the star-forming gas reservoirs (Eq. (8)). Indeed, for a given mass of no-star-forming gas, the rate increases with the dark-matter halo mass, but only up to $M_{\mathrm{h}}=10^{12} M_{\odot}$. Above this threshold the rate is constant for a given mass of no-starforming gas. Thus, for haloes more massive than $M_{\mathrm{h}}=10^{12} M_{\odot}$, the fraction of no-star-forming is increasing.

We also show in Fig. 5 the HI-mass fonction derived by Lagos et al. (2011) using the SAM of Bower et al. (2006). At first order, it is comparable to the mass-function evolution from our reference model $m_{1}$, which is reassuring and expected. It is interesting to note that their predictions for the low-mass range $\left(M<10^{9} M_{\odot}\right)$ are systematically higher than those predicted by our models at $z>0$. This effect could be linked to some resolution effects. At $z=0$, the reference model $m_{1}$ under-predicts the mass function at high mass, while the Lagos et al. (2011) model shows better agreement with the measured HI mass function. This difference can be due to a stronger SMBH feedback in our reference model. Under this hypothesis, in $m_{1}$, with less SMBH feedback, the accretion rate, hence the SFR, would be higher. This would increase the assembled stellar mass and thus the level of stellar mass function that is already too high in the high-mass range.

At $z \simeq 0$, the amount of total gas predicted by $m_{4}$ is larger than the measurement of the HI gas and the lower values of the molecular gas. Independently of each other, the HI and the molecular gas represent only a fraction of the total gas mass contained in a galaxy. However, we can note that for the highmass range, the star-forming gas mass function predicted by $m_{4}$ is in good agreement with the HI mass function measured by 
Table 3. Gas-mass fractions at $z=0$ of (a) HI gas w.r.t total gas in galaxy (gas); (b) star-forming gas (SFG) w.r.t. total gas in galaxy; (c) no-starforming HI gas; (d) (HI-SFG) w.r.t. HI; (e) no star-forming \& no HI gas w.r.t. no star-forming gas; (f) no-star-forming gas w.r.t baryon in the halo.

\begin{tabular}{cccccc}
\hline \hline $\begin{array}{c}\text { HI/gas } \\
\text { (a) }\end{array}$ & $\begin{array}{c}\text { SFG/gas } \\
\text { (b) }\end{array}$ & $\begin{array}{c}\text { noSFG/gas } \\
\text { (c) }\end{array}$ & $\begin{array}{c}\text { (HI-SFG)/HI } \\
\text { (d) }\end{array}$ & $\begin{array}{c}\text { (noSFG-HI)/noSFG } \\
\text { (e) }\end{array}$ & $\begin{array}{c}\text { noSFG/bar } \\
\text { (f) }\end{array}$ \\
\hline 0.084 & 0.025 & 0.975 & 0.70 & 0.91 & 0.65 \\
\hline
\end{tabular}

Zwaan et al. (2005). Even if the total gas mass predicted by $m_{4}$ seems high, without any measurement of this total mass, it is difficult to conclude. The total gas mass function appears today as one of the key observables that will allow us to determine the optimal efficiency of gas ejection process and star formation.

We can integrate at $z=0$ the HI-mass function measured by Zwaan et al. (2005) and the gas-mass function predicted by model $m_{4}$ in the mass range $\left[10^{8}, 10^{12}\right] M_{\odot}$ to compare the gas mass fractions in the different components (Table 3). If we gather the observed and the predicted values, these ratios indicate that:

- only $\simeq 2.5 \%$ of the total gas mass contained in a galaxy can be used directly to form a new generation of stars;

_ $\simeq 70 \%$ of the HI mass should be no-star-forming;

- more than $90 \%$ of the no-star-forming gas is not detected in HI. This fraction is potentially greater than observed, even if its nature still has to be examined in the context of the "missing baryon problem". It could be low-metallicity molecular gas or very hot (X-ray) diluted gas. For instance, some years ago Pfenniger et al. (1994) and Pfenniger \& Combes (1994) proposed that there is a large amount of cold dark gas (essentially in molecular form, $\mathrm{H} 2$ in a fractal structure) evolving in the outer parts of galaxy discs. Following Pfenniger \& Combes (1994), this gas could be no-star-forming and in equilibrium between coalescence, fragmentation, and disruption along a hierarchy of turbulent clumps. In these conditions, the dissipation time of this gas may exceed several Gyr, and only a tiny fraction may be turned into stars. This kind of hidden baryon could be a candidate for a physical explanation of the ad hoc model. As already explained, the total gas mass function appears as a key observable in the context of the new molecular gas surveys.

Comparing models and observations at higher redshift, we see from Fig. 5 that standard models strongly under-predict the amount of observed (molecular) gas, at least for $z \leq 2$ in highmass galaxies. At high masses (dark-matter halo + galaxy), the gas-ejection process is very limited. Thus, the lack of gas mass cannot be explained by an excessive instantaneous ejection in high-mass objects, but by a time-integrated ejection process that is too efficient.

At $z \sim 2$ the amount of molecular gas measured by Berta et al. (2013) is much greater than predicted by all models. Even if the observed gas quantity seems very large, the lack of gas in the models may explain the under-prediction of massive galaxies at these epochs (see Fig. 3).

\subsection{Star formation rate history}

Figures 6 and 7 show the evolution with redshift of the cosmic SFR density (CSFRD) and the stellar mass density, respectively. Models are compared to a set of observational data: Hopkins \& Beacom (2006), Bouwens et al. (2011), and Cucciati et al. (2012) for the SFR density, and Wilkins et al. (2008), Stark et al. (2009), and Labbé et al. (2010) for the stellar mass density. For the sake

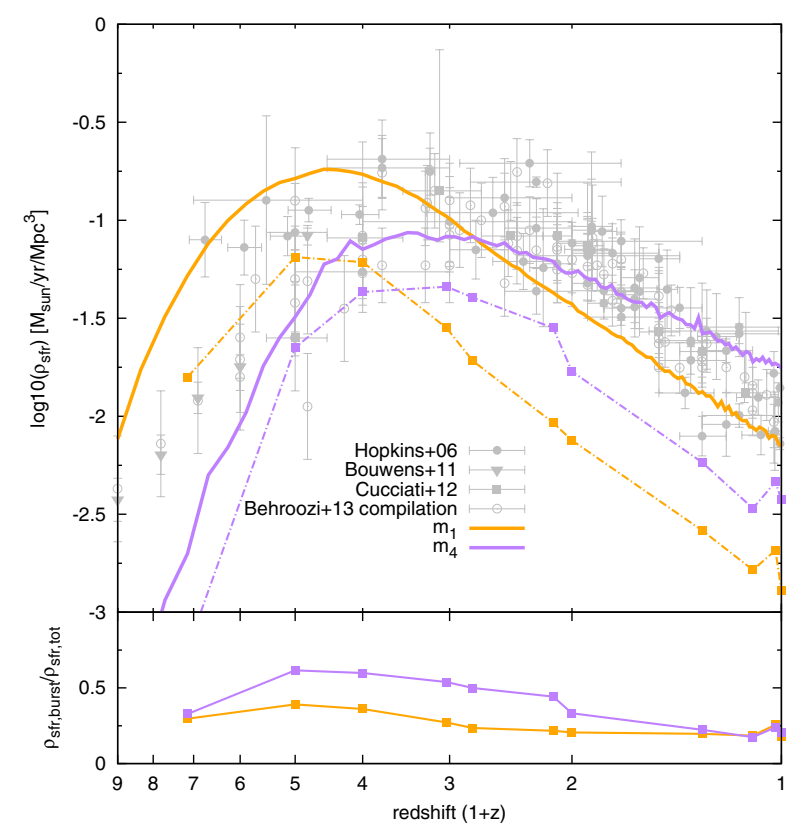

Fig. 6. Redshift evolution of the star formation rate density. Model predictions (coloured lines) are compared with observational data (grey points) coming from Hopkins \& Beacom (2006), Bouwens et al. (2011), and Cucciati et al. (2012). Solid lines present the star formation rates derived from the models. They are computed as the sum of the star formation rate of all galaxies and divided by the box volume $\left((100 / h)^{3} \simeq 150^{3} \mathrm{Mpc}^{3}\right)$. Dashed lines show the merger-driven star formation activity. The bottom panel gives the redshift evolution of the ratio $\rho_{\text {sfr,burt }} / \rho_{\text {sfr,tot }}$. We can see that the fraction of star formation activity linked to merger events is larger in $m_{4}$ than in the reference model $m_{1}$.

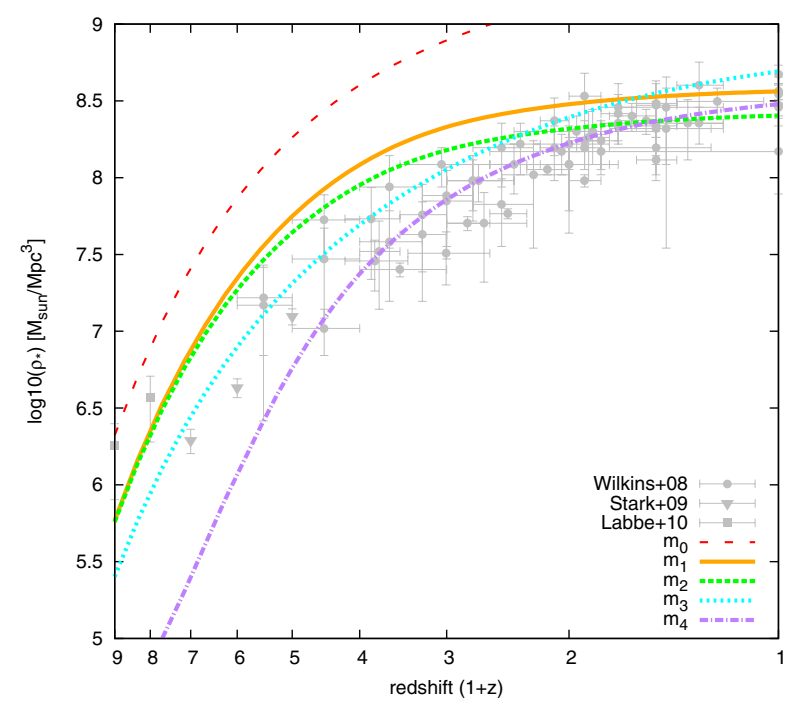

Fig. 7. Redshift evolution of the stellar mass density from the models (coloured lines). Model predictions are compared with observational data (grey points) coming from Wilkins et al. (2008), Stark et al. (2009), and Labbé et al. (2010). 
of clarity, only $m_{1}$ and $m_{4}$ are shown in Fig. 6. Models $m_{2}$ and $m_{3}$ lead to similar evolutions to model $m_{1}$.

Model $m_{1}$, with (SN/AGN)-feedback, presents a peak of CSFRD between $z=3$ and $z=6$ that is marginally compatible with observations. This early peak in star formation is at the origin of the over-production of stellar mass (Fig. 7) in the structures formed at this epoch (with $M_{\text {halo }} \leq 10^{11} M_{\odot}$ ). A strong SFR leads to a strong SN feedback, and consequently to a large amount of mass that definitively leaves the dark matter halo potential $(\simeq 60 \%)$. The gas density in the hot atmosphere becomes too low to produce efficient cooling, and the accretion rates for the galaxies decrease. For example, with our model $m_{1}$ at $z=2$, the mean cooling rate on a $10^{11.5} M_{\odot}$ dark matter halo is $2.15 M_{\odot} \mathrm{yr}^{-1}$. The cooling rate falls at $1.40 M_{\odot} \mathrm{yr}^{-1}$ at $z=0.3$, which is a decrease of $35 \%$. This lack of fresh gas is at the origin of the strong decrease in the SFR found for $m_{1}$ at low redshifts $(z<3)$. Even if the boost factor (Eq. (6)) is applied to the postmerger galaxies, $m_{1}$ cannot correct for this lack of star formation. In the Munich model this decrease is compensated by the reincorporation of the gas previously expelled from the galaxy on a nadapted timescale, which allows increasing the gas mass available to cool.

The redshift evolutions of the CSFRD and stellar mass density predicted by $m_{4}$ are in better agreement with observations. Indeed, the strong reduction of the gas fraction available to form stars allows both reducing the star formation activity at high $z$ and maintaining a larger amount of gas than in model $m_{1}$ at low $z$.

Figure 6 shows, the solid lines the total CSFRD. In the same plot we added a second measurement for information, where we have only taken the merger-driven star-forming galaxies. This kind of galaxy is defined as a post merger structure with a SFR boost (Eq. (6)) leading to SFR values greater than $\overline{S F R}+\sigma_{\mathrm{SFR}}{ }^{5}$. In the steady-state galaxies, the SFR is closely linked to the fresh accretion of gas and is not due to recent merger events. We see from the figure that at high redshift $(z \sim 3)$, in $m_{1}$, the stellar mass growth is dominated by the star formation in steady-state objects. As explained previously, in $m_{4}$ the merger driven star formation process is more efficient than in $m_{1}$. Indeed, even if we apply the merger-boosting factor (Eq. (6)) to the star formation of post-merger galaxies in $m_{1}$, they do not have enough gas to produce large starbursts.

Figure 7 gives a good summary of the situation. We see that for standard models $\left(m_{1}, m_{2}\right.$ and $\left.m_{3}\right)$, the stellar mass is formed too early in the evolution histories of galaxies. Currently, standard SAMs (e.g. Somerville et al. 2008; Guo et al. 2011) are reproducing the local CSFRD and stellar-mass density quite well. But these good results at $z=0$ must not obscure the fact that the history of the stellar mass assembly is not described well. Indeed, as shown in Fig. 7, the stellar mass densities predicted at $z>3$ are systematically larger than the observations. The results obtained at $z=0$ are only due to an excessive decrease in the mean SFR after the strong over-production of stars at high redshift. Indeed, as shown in Fig. 6, for $z<2$ the CSFRD is systematically lower than observed. This decrease is also visible in the slope of the stellar mass density. If the gas is over-consumed at high $z$, it seems to be missing at low $z$. Only the ad hoc model that maintains a large amount of gas in discs allows reproducing at the same time i) the stellar mass density at all redshifts and ii) the trend in the CSFRD.

\footnotetext{
$5 \overline{S F R}$ and $\sigma_{\text {SFR }}$ are respectively the average and the standard deviation of SFR distribution of steady-state galaxies with stellar mass and dark matter halo mass value close (in the same half dex) to the merger-driven starburst galaxy that was considered.
}

\subsubsection{The star formation rate distribution}

Figure 8 shows the SFR distribution function. We compare models $m_{1}, m_{3}$, and $m_{4}$ with a set of observational data. To obtain the SFR distribution we applied the standard conversions from infrared luminosity or ultraviolet magnitude measurements to SFR (Kennicutt 1998 conversions, with a Chabrier IMF, $\left.S F R\left[M_{\odot} \mathrm{yr}^{-1}\right]=3.1 \times 10^{-10} L_{I R(8-1000 \mu \mathrm{m})}\left[L_{\odot}\right]\right)$. In Fig. 8, we are also showing the total and merger-driven SFR. In all cases, the high SFR values are mainly linked to merger events, even if the boost factor generates a wide range of SFR.

As discussed previously, the lack of gas in galaxies formed in $m_{1}$ produces a lower CSFRD than observed at low redshift. As a result, the SFR distribution predicted by $m_{1}$ at low redshift $(0<z<1.8$ in the figure $)$ is always lower than the observations by a factor 0.3 dex on average. For $m_{4}$, the artificial gas concentration allows a large star formation activity to be maintained even if the fraction of star-forming gas is low. This delayed star formation model gives a good match to the observation for $0<z<3$. But this good result must be put inot context. Indeed the strong decrease applied to the star-formation rate in $m_{4}$ leads to a very low level of star formation at high redshift $(z \simeq 6)$, as can be seen in Fig. 8. Even if it seems that we need to strongly reduce the star formation activity if we want to reproduce galaxy properties at low redshift $(z<3)$, the comparison with the Bouwens et al. (2007) measurements indicates that model $m_{4}$ clearly under-predicts the star formation rate at these epochs. The observations show that, in some structures at high redshift (as for Lyman break galaxies), stars are formed with very high efficiency. Also, modelling the high-redshift LymanAlpha emitters, Garel et al. (2012) found that a very high starformation efficiency is needed to reproduce the luminosity function. Our model $m_{4}$ cannot produce these kinds of galaxies.

To reconcile the predicted star formation rate distribution with the observed one, but still producing the same amount of stellar mass in these objects at these epoch ${ }^{6}$, we need to have the same quantity of star-forming gas, but to reduce the dynamical time of the star formation process. To do that we could associate the star-forming gas component to denser regions, with smaller characteristic sizes. This is expected to give star formation rates that would be comparable to those predicted by $m_{1}$. The SFR distribution in $m_{1}$ is in good agreement with Bouwens et al. (2007) at very high $z$, even if the stellar mass produced in these lowmass haloes is higher than observed. In this model, the rhythm of star formation is good, but the star formation acts on gas reservoirs that are too large. The star formation activity produced by $m_{3}$ (based on Somerville et al. $2008 \mathrm{SN}$-feedback), compared to the reference model $m_{1}$, under-predicts this high-redshift starforming population. This is probably due to the strong photoionization and $\mathrm{SN}$-feedback processes considered in this model.

Measurements of star formation rate, given by Reddy et al. (2008) and Bouwens et al. (2007), are computed using UV observations, corrected from dust extinction. This correction is based on the UV continuum $/ \beta$-slope extinction law and is mainly an extrapolation of results from local galaxies (e.g. Burgarella et al. 2005). Even if this relation seems to be valid at redshift $z \simeq 2$ (e.g. Daddi et al. 2007; Reddy et al. 2012), it has a huge scatter, and extrapolations to large redshifts lead to large errors (especially at high SFR) that are difficult to estimate.

For completeness, we added the distribution function of the filamentary accretion rate to Fig. 8 and the cooling rate for $m_{4}$. As expected, the cold mode efficiency decreases when redshift

\footnotetext{
6 Indeed, as shown in the stellar mass function, the build-up of the stellar mass agrees with the observed stellar mass function.
} 
M. Cousin et al.: Galaxy stellar mass assembly

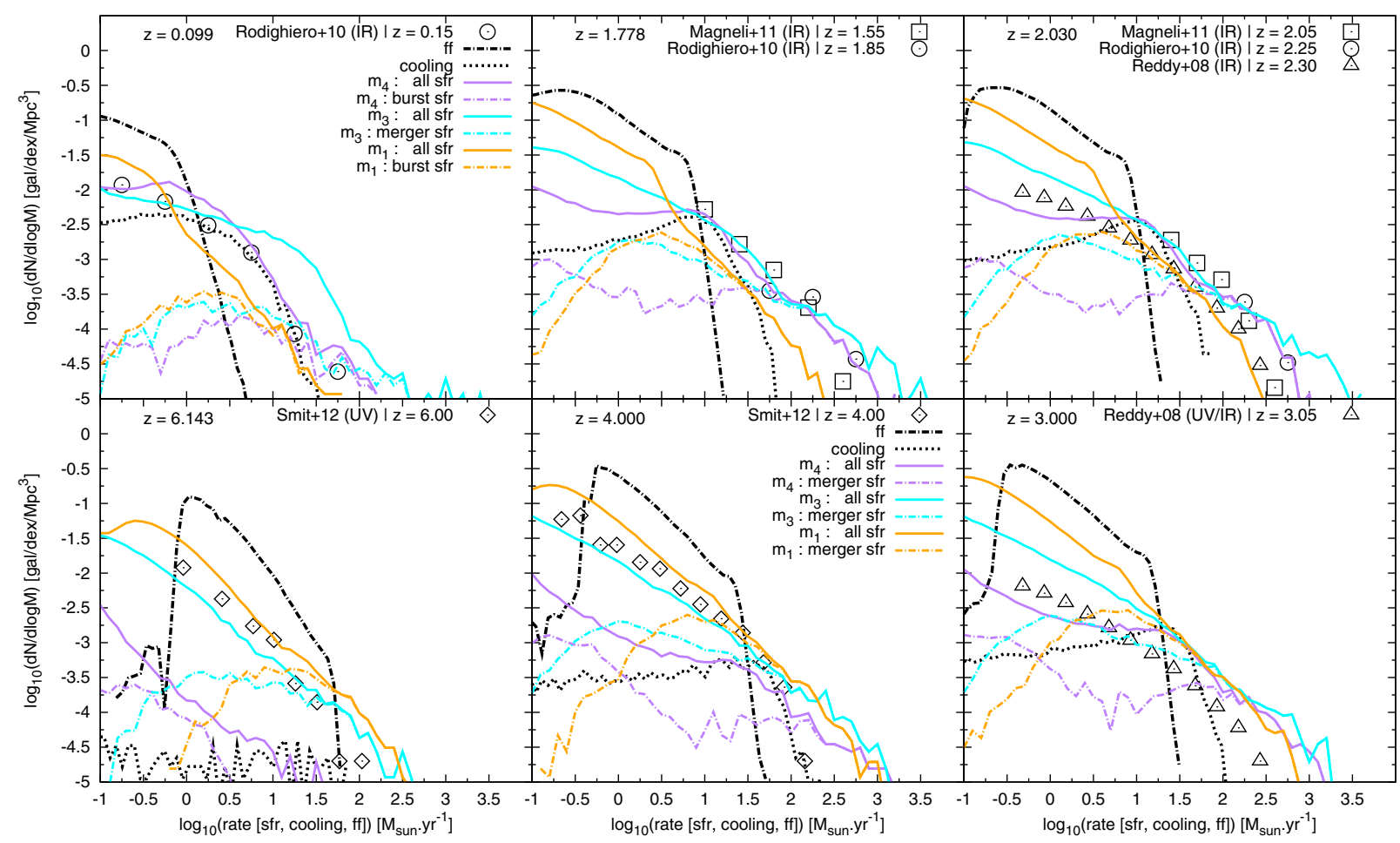

Fig. 8. Star formation rate distribution for $m_{1}, m_{3}$, and $m_{4}$. Solid and dot-dashed coloured lines give the total and the merger-induced (Eq. (6)) star formation rate distribution, respectively. The merger-induced distributions are built with galaxies that i) have merged during the last time-step and ii) have $S F R>\overline{S F R}+\sigma_{\mathrm{SFR}}$, where $\overline{S F R}$ and $\sigma_{S F R}$ are, respectively, the mean and standard deviation of star formation rate distribution of steady-state galaxies with stellar mass and dark matter halo mass value close (in the same half dex) to the merger-driven starburst galaxy taken into account. Models are compared with a set of observational data coming from Rodighiero et al. (2010; circles), Magnelli et al. (2011; squares) and Reddy et al. (2008; triangles) at low redshift $(z \leq 3)$, and from Smit et al. (2012; diamonds) at high redshift $(z=4-6)$. For information we add the free-fall rate (black dash-dot line) and cooling rate (black dot line) distribution function for model $m_{4}$.

decreases. At high SFR, it is interesting to note that no galaxy is accreting enough baryons to form stars in a steady-state mode; only the episodic merger events can produce these high values.

\subsubsection{The specific star formation rate}

Figure 9 shows the sSFR. We compare all models with a set of observations from Dunne et al. (2009), Karim et al. (2011), Gonzalez et al. (2012), Reddy et al. (2012) and with Béthermin et al. (2012) model predictions. First, for all models we note that the mean sSFR (over the whole mass range) increases with redshift. More specifically, we show in Fig. 10 the redshift evolution of the sSFR, extracted from model $m_{4}$, for two mass ranges. We see that more massive galaxies have lower sSFR at any redshift, implying that they form the bulk of their stars earlier than their low-mass counterparts.

At all redshift $z \leq 3$ and for the whole mass range, models $m_{1}$ and $m_{2}$ are systematically lower (by a factor 10) than the observations. This result is due, on the one hand, to the low SFR as seen in Fig. 8, and on the other to the excess of stellar mass as seen in Fig. 4.

The model with stronger SN-feedback $\left(m_{3}\right)$ gives better results in the intermediate redshift and mass ranges $(0<z<3$; $10^{10}<M_{\star}<10^{11} M_{\odot}$ ) but stays lower than the observations in the lower mass regime for the same reasons as explained previously. The predictions at high mass suffer from the absence of AGN-feedback and do not have to be considered.

Model $m_{4}$ gives better agreement with the observations than the reference model $m_{1}$ or its variation $m_{2}$ does. In $m_{4}$, the mean star-formation efficiency is higher. This result does not contradict with the main objective of this ad-hoc model. The star formation activity is strongly reduced, and thus the produced stellar mass is also strongly reduced. This two trends lead to a higher level of the sSFR than in $m_{1}$ and $m_{2}$.

Figure 11 shows the sSFR distributions of galaxies predicted by model $m_{4}$ in a limited stellar mass range such that they can be directly compared with the Sargent et al. (2012) observational measurements. The population of quiescent galaxies has been removed in Sargent et al. (2012), we added two log-normal distributions to our histograms to allow for a better comparison:

$$
\begin{aligned}
& \text { - a first one fit the MS population; } \\
& \text { - a second one fit the merger-driven population. }
\end{aligned}
$$

The excess of objects at low sSFR in the histograms, in comparison to the MS log-normal distribution, corresponds to the quiescent galaxy population.

We can see that the MS distribution predicted by $m_{4}$ is in excellent agreement with Sargent et al. (2012). However, the number of galaxies in the PMSB mode is over-predicted even if the average values of the distribution is in good agreement with what is observed $\left(\log _{10}\left(s S F R / S F R_{\mathrm{MS}}\right) \simeq 0.6\right)$. It is possible to reduce this tension by modifying our PMSB definition $\left(S F R_{\mathrm{PMSB}} \geq \overline{S F R}+\sigma_{\mathrm{SFR}}\right)$. If we replace $\sigma_{\mathrm{SFR}}$ by $2 \sigma_{\mathrm{SFR}}$ in the previous definition, the number of objects on the PMSB population obviously decreases and becomes comparable to Sargent et al. (2012), but in this case, the centroid of the PMSB distribution is then shifted to a higher value $\left(\log _{10}\left(s S F R / S F R_{\mathrm{MS}}\right) \simeq 1\right)$. The properties of the PMSB population are also strongly dependent of the merger-boost factor used in the model (Eq. (6)). 


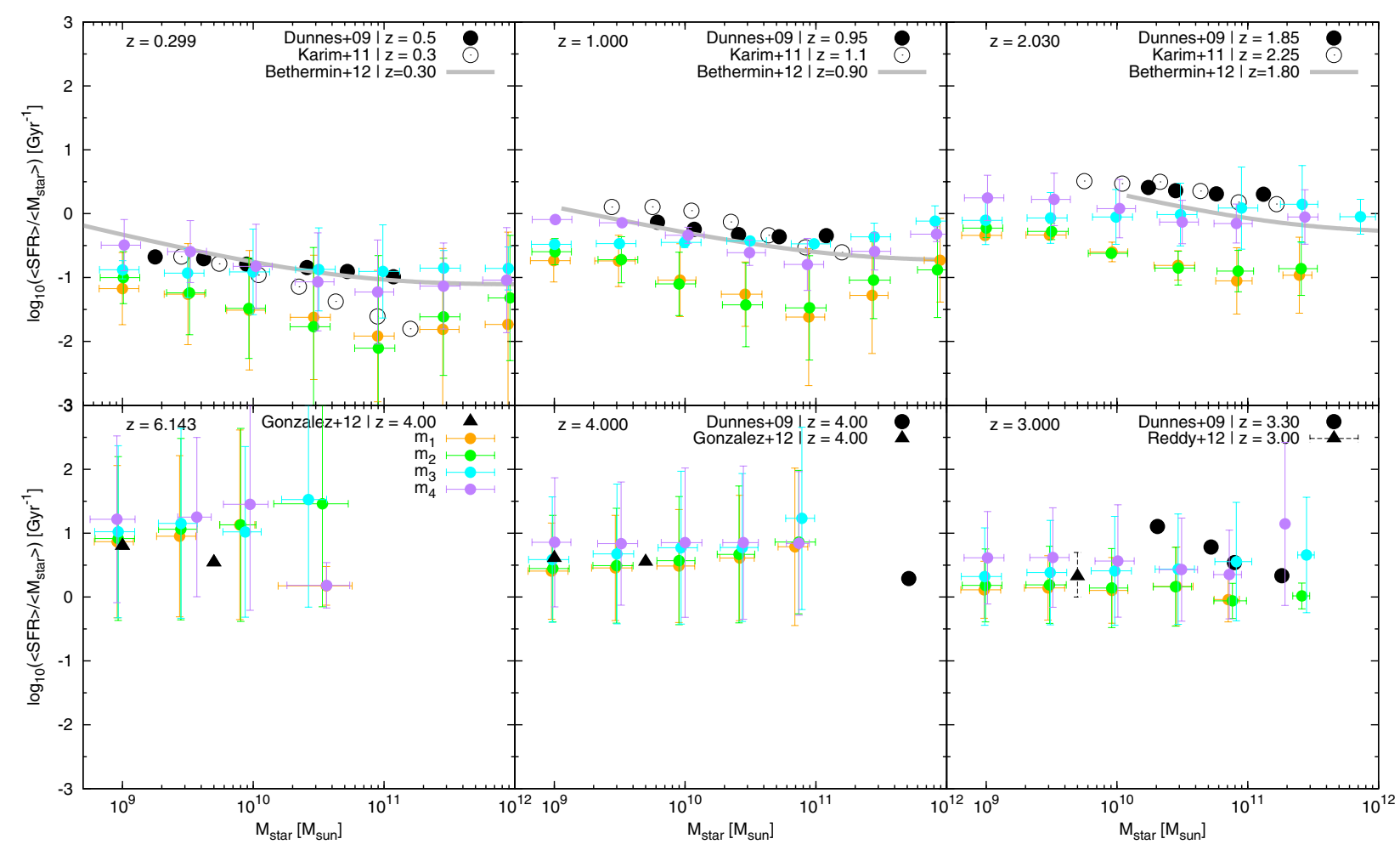

Fig. 9. Specific star formation rate as a function of the stellar mass for a set of redshifts (the redshift is increasing clockwise). This quantity can be seen as the inverse of the stellar mass doubling time. The higher the value, the more intense the star formation activity. We compare the models (colour-coded) with observational data from Dunne et al. (2009), Karim et al. (2011), Gonzalez et al. (2012), Reddy et al. (2012), and Béthermin et al. (2012). For most observational data points, errors are smaller than the size of the symbol.

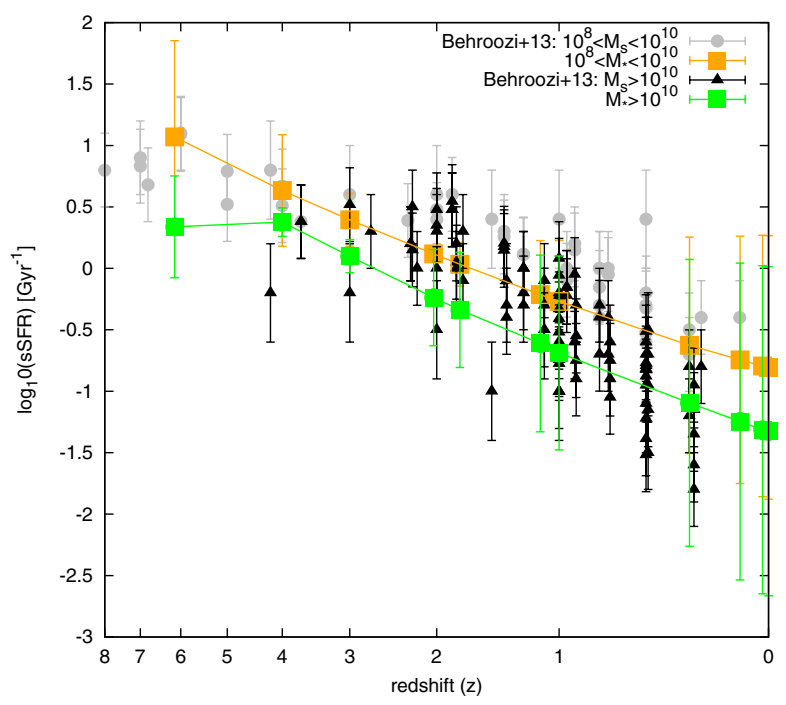

Fig. 10. Redshift evolution of the specific star formation rate derived from $m_{4}\left(10^{8}<M_{\star}<10^{10} M_{\odot}\right.$ in orange and $10^{10}<M_{\star}<10^{12} M_{\odot}$ in green). The error bars correspond to the standard deviation. For comparison, we show the data points around $M_{\star}=10^{9} M_{\odot}$ and $M_{\star}=10^{10} M_{\odot}$, extracted from Behroozi et al. (2013b).

Some modifications, such as on the amplitude of the characteristic merger time scale $\left(\tau_{\text {merger }}\right)$, will be explored in a future work.

\subsection{Steady-state versus merger-driven star formation}

To summarize, galaxies evolve in a quasi-steady state in $m_{1}$, $m_{2}$, and $m_{3}$, after the high level of star formation activity at $4<z<6$, and consequently the high rates of mass ejection. The star-formation rate is directly proportional to the gas-accretion rate. At intermediate mass $\left(M_{\mathrm{h}} \simeq 10^{11} M_{\odot}\right)$, there is not enough hot gas in equilibrium in the dark-matter potential well (owing to the strong feedback), and therefore the cooling is not efficient. Consequently, these intermediate-mass galaxies are deficient in fresh gas, and the star-formation rate becomes lower than observed at $1<z<3$ (Figs. 8 and 6). In Guo et al. (2011) and Henriques et al. (2013), the lack of gas in the hot phase is compensated for by the reincorporation of the gas ejected previously that was stored in a passive reservoir.

In the delayed star formation model $m_{4}$, the amount of the star formation rate occurring in merger events and amplified by the boost factor is higher than in $m_{1}$ (see Fig. 8). Indeed, mergers generate a strong increase in the star formation activity because of the large amount of the accreted gas that is in the disc in the no-star-forming phase and to the merger-induced no-starforming to star-forming gas conversion (Eqs. (8) and (6)). The rapid transformation of gas into stars allows reaching very high star-formation rates (see Fig. 8). At lower redshift, as in standard models, the larger amount of stellar mass is formed in the quasi-steady state mode. Even if, at these epochs, the contribution of merger events strongly decreases, the highest star formation rates are still found in post-merger structures.

\section{Discussion and conclusion}

We have presented four galaxy formation models and compared them. We showed that classical models $m_{1}$ (reference), $m_{2}$, and $m_{3}$ fail to reproduce the faint end of the stellar-mass function. They over-predict the stellar mass in the low-mass dark matter haloes $\left(M_{\mathrm{h}}<10^{10} M_{\odot}\right)$. Even when a strong photoionization 
M. Cousin et al.: Galaxy stellar mass assembly

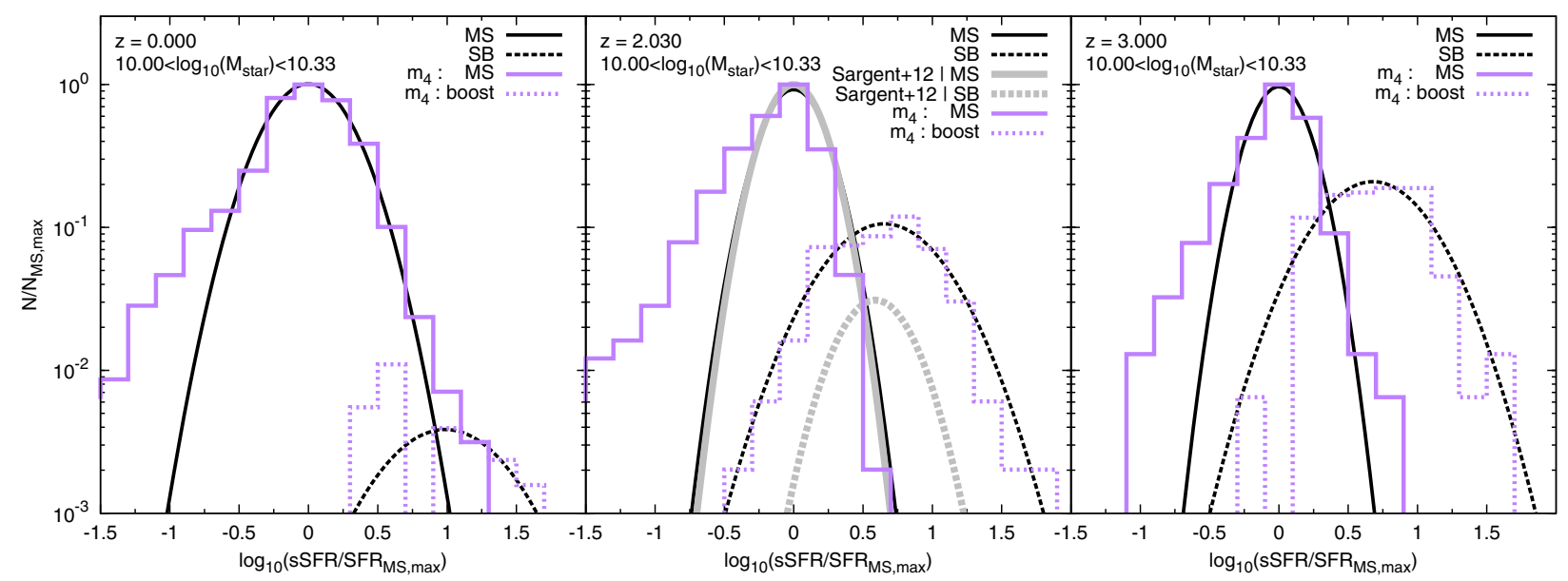

Fig. 11. Specific star formation rate distributions derived from model $m_{4}$, for galaxies with a stellar mass in the range: $10<\log _{10}\left(M_{\star}\right)<10.33$. The purple solid histogram shows the distribution of galaxies in the steady state mode ( $S F R \propto$ accretion rate). Such a distribution contains main sequence (MS) and quiescent galaxies $\left(s S F R / S F R_{\mathrm{MS}}<0.1\right.$ in our study). The purple dashed-line histogram shows the distribution of the sSFR for galaxies with post-merger star-formation activity (PMSB) (Eq. (6) and following $S F R_{\mathrm{PMSB}} \geq \overline{S F R}+\sigma_{\mathrm{SFR}}$ ). At $z=2$ we compare our results with Sargent et al. (2012) measurements in which the quiescent population have been removed (grey solid line). For a better comparison with these observational measurements, we add a log-normal distribution as a black solid line adjusted only on our MS galaxy population. We have removed all galaxies with $S S F R / S F R_{\mathrm{MS}}<0.1$. We see that model $m_{4}$ is in very good agreement with the MS distribution but over-predicts the number of galaxy in the post-merger starburst mode $(0.4 \mathrm{dex})$.

and $\mathrm{SN}$-feedback are used (as in $m_{2}$ and $m_{3}$ ), the models form too many stars in the low-mass range. Moreover, recent observations indicate that the loading factors $\left(\dot{M}_{\mathrm{ej}} / \dot{M}_{\star}\right)$ are much smaller that those predicted by such models. A strong SN-feedback generates a strong decrease in the amount of gas, which has to be compensated for at low $z$, for example, by reincorporating some gas (Henriques et al. 2013). Such a problem in the low-mass structures is invariably present, even in the most recent SAMs (Guo et al. 2011; Bower et al. 2012; Weinmann et al. 2012) and, as explained by Henriques et al. (2013), can thus be viewed as a generic problem.

On the basis of a comparison between the models for which the stellar-mass functions and the relationship between $M_{\mathrm{h}}$ and $M_{\star}$ are reproduced quite well at $z=0$ (e.g. Guo et al. 2011), it seems that the problem occurs at high redshift. But, if the relations at high $z$ are not reproduced, then the history of the structures populating the relations at lower redshift is not consistent, even if the stellar-mass functions at these low redshifts seem agrees with observations.

If we consider that the $\Lambda$-CDM paradigm produces the correct number of low-mass dark matter haloes, then the star formation activity has to be strongly reduced in this range of masses to reproduce the observations. We applied this condition in an ad-hoc model $\left(m_{4}\right)$. This model is based on a two-phase gaseous disc with, on the one hand the star-forming gas, and on the other, the no-star-forming gas. We showed that $m_{4}$ is in good agreement with a large set of observations, even if there is a tension with the $z>4$ SFR computed from UV measurements (Bouwens et al. 2007).

A galaxy formation and evolution model that uses a strong modification of the star formation activity (quenched or limited) has already been studied by Bouché et al. (2010). In their model, the fresh gas accretion is halted for haloes with masses lower than $M_{\mathrm{h}}=10^{11} M_{\odot}$. In our model, such small structures host a very large amount of no-star-forming gas. That this gas has been accreted but cannot form star leads to the same result as the nonaccretion model proposed by Bouché et al. (2010). The results, produced by the toy model described in Bouché et al. (2010), are in good agreement with observations (sSFR, their Fig. 4, and the Tully-Fisher relation, their Fig. 5). This independent study reinforces the hypothesis of a strong decrease in the star formation efficiency in low-mass structures at high redshift.

In classical SAMs, the cold gas that can form star is modelled as a homogeneous component, generally following a decreasing radial exponential profile. In addition, these models consider that a given fraction of the gas is available to form stars at any moment. The SFR applied to this cold gas reservoir is computed following Kennicutt (1998) empirical law, without knowing the exact fraction of the gas that can actually be impacted by the process. The ad hoc model presented in this paper is based on a no-star-forming gas component that can be seen as the gaseous fraction that is not in optimal conditions forming stars, i.e., not above the critical density threshold.

Observations show that only a small fraction of the total gas mass $(\simeq 15 \%)$ is above the optimal column density threshold, and only a small fraction $(\simeq 15 \%)$ of this dense gas is in prestellar cores (André 2013; André et al. 2014). Indeed, before being in the form of a prestellar core, the gas in the ISM must follow a continuous structuration process, from the low density accreted gas to the highest density regions. This structuration cascade needs time and obviously, at a given time, all the gas in the disc cannot be available to form stars. The no-star-forming to star-forming conversion process may be linked to the global dynamic of the disc. Indeed in highly-disturbed discs (with a $V / \sigma_{\mathrm{v}} \in[1-10]$ ), as observed at high $z$ (e.g. Genzel et al. 2006, 2008; Stark et al. 2008; Cresci et al. 2009) and seen in hydrodynamic simulations (e.g. Kereš et al. 2005; Dekel et al. 2009a,b; Khochfar \& Silk 2009), star formation occurs only in a few highdensity regions (clumps).

In addition to these large scale disturbed dynamics at high $z$, the density structuration process can be limited by other mechanisms that are seen at low $z$. The turbulence heating can be one of them. Even if the main driver of this turbulence is not clearly understood (shocks, SN kinetic energy injection, tidal interactions, galaxy collisions), recent infrared spectroscopic observations at intermediate redshift $(1<z<2)$ show that the molecular 
gas can be dynamically heated by turbulence (e.g. Guillard et al. 2009, 2012; Ogle et al. 2010; Appleton et al. 2013) and is thus not available for star formation. Hydrodynamic simulations by Bournaud et al. (2010) also show that the SN energy injection disrupts the dense regions on the smallest scales (ten to hundred parsecs) from the typical size of star-forming filaments in the ISM to a typical disc-scale height at $z \simeq 2$.

The main idea behind the no-star-forming gas reservoir is that, at any given time, only a fraction of the gas can form stars. Indeed even if Kennicutt's law gives a relation between the gas content (mass), the geometry (galaxy size), and the star formation activity, it does not give any information about the gas fraction that is turned into stars. In a galaxy, a set of highly concentrated star-forming regions that affect a small amount of the total gas mass can be compatible with the Kennicutt's law (sum of SFR in all regions). However, our ad-hoc model $m_{4}$ leads to a fraction of no-star-forming gas at $z=0$ that is greater than given by observations. Our analysis can also be compared to previous works that introduced a significant fraction of gas that cannot form stars (e.g. Pfenniger et al. 1994; Pfenniger \& Combes 1994). In this context, the study of the gas dynamics and states (with ALMA for instance) will be a key point in understanding the regulation of star formation in galaxies.

To model the two-phase disc, we assumed that the accreted gas is composed of a large fraction (99\%) of no-star-forming gas, that is progressively converted in the star-forming phase. The conversion rate (Eq. (8)) has not been defined to explicitly and physically follow the structuration process but is calibrated to reproduce the SHMR (e.g. Leauthaud et al. 2012; Moster et al. 2010; Behroozi et al. 2010; Béthermin et al. 2012). This formulation has no other purpose than to highlight the order of magnitude of the regulation process that has to be introduced. To reproduce Kennicutt's law on a galaxy scale, even when a large fraction of the gas can not form stars, we have artificially concentrated the star-forming gas in the centre of the disc where the density is the highest. This ad-hoc modification leads to very good results mainly for the stellar-mass functions and sSFR. On the other hand, the SFR distribution predicted by this model strongly disagrees with SFR measurements at $z>4$. It shows the need for an explicit description of the density structuration process. The gas-mass function predicted by the ad-hoc model may indicate that galaxies have a gas content that is too large, even if the comparison with observations is difficult because the total gas mass function is not known. In the future measuring the gas mass function will be a key observable that will constrain the balance between the ejection process and gas regulation in galaxies.

In a forthcoming work, we plan to follow the structuration of the gas, using a semi-analytical approach from the largest scales ( $r>h$, the disc-scale height), where the dynamics are governed by (quasi-)2D turbulence, to the smallest scales $(r<h)$, where the star formation process occurs in $3 \mathrm{D}$ molecular clouds (Romeo et al. 2010; Bournaud et al. 2010). In current SAMs, the SFR is only linked to the gas component and to the overall galaxy dynamics $\left(t_{\mathrm{dyn}}\right)$. This approach is not able to correctly describe the regulation of the star formation. Galaxy discs are complex structures based on multi-fluid interactions on multiscales (2D, 3D; Shi et al. 2011). The connexions between the stellar and gas components generate instabilities that participate in the ISM structuration (e.g. Jog \& Solomon 1984; Hoffmann $\&$ Romeo 2012). Star formation and disc instabilities are thus strongly linked. A better description of these ISM structuration mechanisms is essential for a better understanding of the star formation regulation processes.
Acknowledgements. The authors thank Jean-loup Puget and Pierre Guillard for very useful comments and discussions. We also thank the referee for a very helpful report. We acknowledge financial support from the "Programme National de Cosmologie and Galaxies" (PNCG) of CNRS/INSU, France.

\section{Appendix A: Cosmic variance}

For the sake of clarity, errors bars due to cosmic variance in the stellar mass function are not plotted in Fig. 3. We have indicated a threshold above which uncertainties become larger than the variations observed for the different models. For illustration, we show in Fig. A.1 the stellar mass functions predicted by the models at $z=0.3$, where we have added the error bars linked to the cosmic variance.

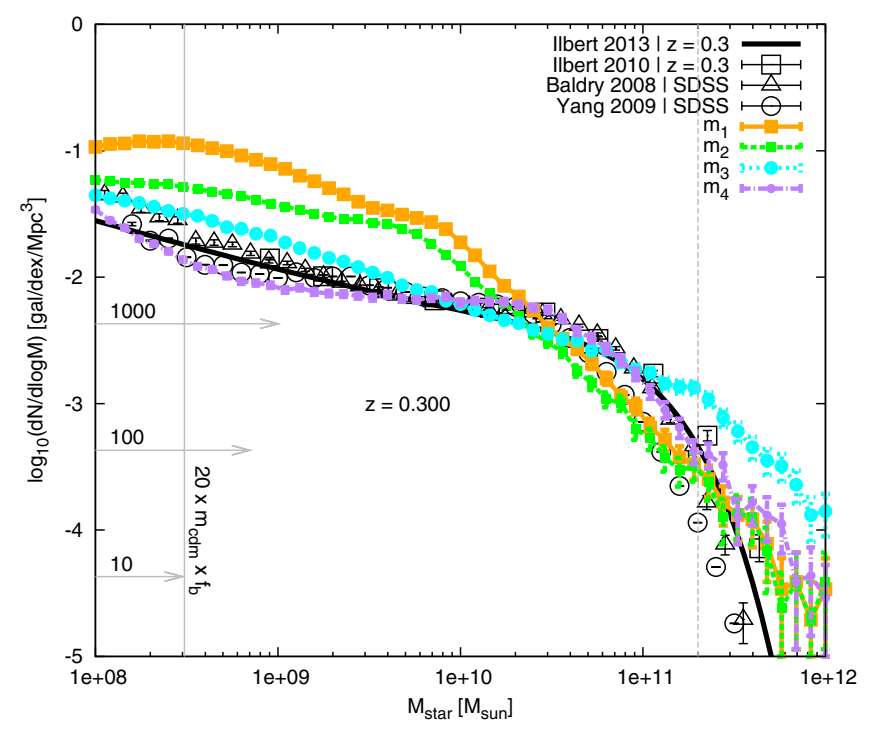

Fig. A.1. Stellar mass function predicted by our different models at $z=$ 0.3 . The colour code is given in Table 1 . We compare our results with Ilbert et al. (2010, 2013; squares), Yang et al. (2009; circles) and Baldry et al. (2008; triangles) observations. Horizontal arrows show the link between the density and the number of haloes in our simulation volume. We show in this figure the error bars related to cosmic variance.

\section{Appendix B: The photoionization filtering masses}

We used two different models of photoionization based on two definitions of the filtering mass. We recall here the expressions that we have used.

\section{B.1. Prescription for $m_{1}$}

For the Okamoto et al. (2008) model, we used an expression deduced from a fit of the filtering mass evolution given in their Fig. 15:

$M_{\mathrm{c}}(z)=6 \times 10^{9} h_{0}^{-1} \exp (-0.7 z) M_{\odot}$.

\section{B.2. Prescription for $m_{2}$}

In Gnedin (2000) and Kravtsov et al. (2004), the filtering mass is defined as a function of the expansion factor $(a)$ :

$M_{\mathrm{c}}(a)=2.5 \times 10^{11} h^{-1} \Omega_{\mathrm{m}}^{-1 / 2} \mu^{-3 / 2} f(a) M_{\odot}$ 
where $f(a)$ is the conditional function given in Eq. (B.3). As explained in Kravtsov et al. (2004), the best agreement with hydrodynamic simulations is obtained with $\alpha=6$.

$$
f(a)= \begin{cases}\frac{3 a}{(2+\alpha)(5+2 \alpha)\left(\frac{a}{a_{0}}\right)^{\alpha}} & \text { if } a \leq a_{0} \\ \frac{3}{a}\left[a_{0}^{2}\left[\frac{1}{2+\alpha}-\frac{2\left(a / a_{0}\right)^{-1 / 2}}{5+2 \alpha}\right]+\frac{a^{2}}{10}\right. & \\ \left.-\frac{a_{0}^{2}}{10}\left[5-4\left(a / a_{0}\right)^{-1 / 2}\right]\right] & \text { if } a_{0} \leq a \leq a_{r} \\ \frac{3}{a}\left[a_{0}^{2}\left[\frac{1}{2+\alpha}-\frac{2\left(a / a_{0}\right)^{-1 / 2}}{5+2 \alpha}\right]+\frac{a_{r}^{2}}{10}\left[5-4\left(a / a_{r}\right)^{-1 / 2}\right]\right. & \\ \left.-\frac{a_{0}^{2}}{10}\left[5-4\left(a / a_{0}\right)^{-1 / 2}\right]+\frac{a a_{r}}{3}-\frac{a_{r}^{2}}{3}\left[3-2\left(a / a_{r}\right)^{-1 / 2}\right]\right] & \text { if } \geq a_{r} .\end{cases}
$$

\section{Appendix C: Data repository}

Outputs from all models are available at the CDS. Data are distributed under *. fits format and are therefore compatible with the TOPCAT software (http: //www.star.bris.ac.uk/ mbt/ topcat/).

\section{References}

André, P. 2013 [arXiv: 1309.7762]

André, P., Men'shchikov, A., Bontemps, S., et al. 2010, A\&A, 518, L102

André, P., Di Francesco, J., Ward-Thompson, D., et al. 2014, in Protostars and

Planets VI, eds. H. Beuther, R. Klessen, C. Dullemond, \& Th. Henning

(University of Arizona Press), accepted [arXiv: 1312.6232]

Appleton, P. N., Guillard, P., Boulanger, F., et al. 2013, ApJ, accepted [arXiv: 1309.1525]

Babul, A., \& Rees, M. J. 1992, MNRAS, 255, 346

Baldry, I. K., Glazebrook, K., \& Driver, S. P. 2008, MNRAS, 388, 945

Baugh, C. M. 2006, Rep. Prog. Phys., 69, 3101

Behroozi, P. S., Conroy, C., \& Wechsler, R. H. 2010, ApJ, 717, 379

Behroozi, P. S., Wechsler, R. H., \& Conroy, C. 2013a, ApJ, 762, L31

Behroozi, P. S., Wechsler, R. H., \& Conroy, C. 2013b, ApJ, 770, 57

Bell, E. F., Zheng, X. Z., Papovich, C., et al. 2007, ApJ, 663, 834

Benson, A. J., Lacey, C. G., Baugh, C. M., Cole, S., \& Frenk, C. S. 2002, MNRAS, 333, 156

Berta, S., Lutz, D., Nordon, R., et al. 2013, A\&A, 555, L8

Bertone, S., Stoehr, F., \& White, S. D. M. 2005, MNRAS, 359, 1201

Béthermin, M., Doré, O., \& Lagache, G. 2012, A\&A, 537, L5

Blumenthal, G. R., Faber, S. M., Primack, J. R., \& Rees, M. J. 1984, Nature, 311,517

Blumenthal, G. R., Faber, S. M., Flores, R., \& Primack, J. R. 1986, ApJ, 301, 27 Bondi, H. 1952, MNRAS, 112, 195

Bouché, N., Dekel, A., Genzel, R., et al. 2010, ApJ, 718, 1001

Bouché, N., Hohensee, W., Vargas, R., et al. 2012, MNRAS, 426, 801

Bournaud, F., Elmegreen, B. G., \& Elmegreen, D. M. 2007, ApJ, 670, 237

Bournaud, F., Daddi, E., Elmegreen, B. G., et al. 2008, A\&A, 486, 741

Bournaud, F., Elmegreen, B. G., Teyssier, R., Block, D. L., \& Puerari, I. 2010,

MNRAS, 409, 1088

Bouwens, R. J., Illingworth, G. D., Franx, M., \& Ford, H. 2007, ApJ, 670, 928

Bouwens, R. J., Illingworth, G. D., Oesch, P. A., et al. 2011, ApJ, 737, 90

Bower, R. G., Benson, A. J., Malbon, R., et al. 2006, MNRAS, 370, 645

Bower, R. G., Benson, A. J., \& Crain, R. A. 2012, MNRAS, 422, 2816

Brinchmann, J., Charlot, S., White, S. D. M., et al. 2004, MNRAS, 351, 1151

Bullock, J. S., Kravtsov, A. V., \& Weinberg, D. H. 2000, ApJ, 539, 517

Burgarella, D., Buat, V., \& Iglesias-Páramo, J. 2005, MNRAS, 360, 1413

Capelo, P. R., Coppi, P. S., \& Natarajan, P. 2012, MNRAS, 422, 686

Caputi, K. I., Cirasuolo, M., Dunlop, J. S., et al. 2011, MNRAS, 413, 162

Ceverino, D., Dekel, A., \& Bournaud, F. 2010, MNRAS, 404, 2151

Ceverino, D., Dekel, A., Mandelker, N., et al. 2012, MNRAS, 420, 3490

Chabrier, G. 2003, PASP, 115, 763

Chary, R., \& Elbaz, D. 2001, ApJ, 556, 562

Cole, S. 1991, ApJ, 367, 45
Cole, S., Aragon-Salamanca, A., Frenk, C. S., Navarro, J. F., \& Zepf, S. E. 1994, MNRAS, 271, 781

Cole, S., Lacey, C. G., Baugh, C. M., \& Frenk, C. S. 2000, MNRAS, 319, 168

Conroy, C., \& Wechsler, R. H. 2009, ApJ, 696, 620

Couchman, H. M. P., \& Rees, M. J. 1986, MNRAS, 221, 53

Cousin, M., Lagache, G., Béthermin, M., \& Guiderdoni, B. 2015, A\&A, 575, A33

Cowie, L. L., Hu, E. M., \& Songaila, A. 1995, AJ, 110, 1576

Cresci, G., Hicks, E. K. S., Genzel, R., et al. 2009, ApJ, 697, 115

Croton, D. J., Springel, V., White, S. D. M., et al. 2006, MNRAS, 365, 11

Cucciati, O., Tresse, L., Ilbert, O., et al. 2012, A\&A, 539, A31

Daddi, E., Dickinson, M., Morrison, G., et al. 2007, ApJ, 670, 156

Daddi, E., Bournaud, F., Walter, F., et al. 2010, ApJ, 713, 686

De Lucia, G., \& Blaizot, J. 2007, MNRAS, 375, 2

Dekel, A., \& Silk, J. 1986, ApJ, 303, 39

Dekel, A., Birnboim, Y., Engel, G., et al. 2009a, Nature, 457, 451

Dekel, A., Sari, R., \& Ceverino, D. 2009b, ApJ, 703, 785

Doroshkevich, A. G., Zel'dovich, Y. B., \& Novikov, I. D. 1967, Sov. Ast., 11, 233

Dunne, L., Ivison, R. J., Maddox, S., et al. 2009, MNRAS, 394, 3

Dutton, A. A., \& van den Bosch, F. C. 2009, MNRAS, 396, 141

Efstathiou, G. 1992, MNRAS, 256, 43P

Elbaz, D., Dickinson, M., Hwang, H. S., et al. 2011, A\&A, 533, A119

Elmegreen, B. G. 2009, in Galaxy Evolution: Emerging Insights and Future Challenges, eds. S. Jogee, I. Marinova, L. Hao, \& G. A. Blanc, ASP Conf. Ser., 419, 23

Elmegreen, B. G., \& Elmegreen, D. M. 2005, ApJ, 627, 632

Faucher-Giguère, C.-A., Kereš, D., \& Ma, C.-P. 2011, MNRAS, 417, 2982

Frye, B., Broadhurst, T., \& Benítez, N. 2002, ApJ, 568, 558

Fu, L., Semboloni, E., Hoekstra, H., et al. 2008, A\&A, 479, 9

Garel, T., Blaizot, J., Guiderdoni, B., et al. 2012, MNRAS, 422, 310

Genzel, R., Tacconi, L. J., Eisenhauer, F., et al. 2006, Nature, 442, 786

Genzel, R., Burkert, A., Bouché, N., et al. 2008, ApJ, 687, 59

Gispert, R., Lagache, G., \& Puget, J. L. 2000, A\&A, 360, 1

Gnedin, N. Y. 2000, ApJ, 542, 535

Gonzalez, V., Bouwens, R., llingworth, G., et al. 2012 [arXiv: 1208.4362]

Granato, G. L., Lacey, C. G., Silva, L., et al. 2000, ApJ, 542, 710

Gruppioni, C., Pozzi, F., Andreani, P., et al. 2010, A\&A, 518, L27

Guiderdoni, B., Bouchet, F. R., Puget, J.-L., Lagache, G., \& Hivon, E. 1997, Nature, 390, 257

Guillard, P., Boulanger, F., Pineau Des Forêts, G., \& Appleton, P. N. 2009, A\&A, 502,515

Guillard, P., Boulanger, F., Pineau des Forêts, G., et al. 2012, ApJ, 749, 158

Guo, Q., White, S., Boylan-Kolchin, M., et al. 2011, MNRAS, 413, 101

Hatton, S., Devriendt, J. E. G., Ninin, S., et al. 2003, MNRAS, 343, 75

Heckman, T. M., Lehnert, M. D., Strickland, D. K., \& Armus, L. 2000, ApJS, 129,493

Heiderman, A., Evans, II, N. J., Allen, L. E., Huard, T., \& Heyer, M. 2010, ApJ, 723, 1019

Henriques, B. M. B., White, S. D. M., Thomas, P. A., et al. 2013, MNRAS, 431, 3373

Hernquist, L. 1990, ApJ, 356, 359

Hoeft, M., Yepes, G., Gottlöber, S., \& Springel, V. 2006, MNRAS, 371, 401

Hoekstra, H., Yee, H. K. C., \& Gladders, M. D. 2004, ApJ, 606, 67

Hoffmann, V., \& Romeo, A. B. 2012, MNRAS, 425, 1511

Hopkins, A. M., \& Beacom, J. F. 2006, ApJ, 651, 142

Ikeuchi, S. 1986, Ap\&SS, 118, 509

Ilbert, O., Salvato, M., Le Floc'h, E., et al. 2010, ApJ, 709, 644

Ilbert, O., McCracken, H. J., Le Fevre, O., et al. 2013, A\&A, 556, A55

Jog, C. J., \& Solomon, P. M. 1984, ApJ, 276, 114

Karim, A., Schinnerer, E., Martínez-Sansigre, A., et al. 2011, ApJ, 730, 61

Kauffmann, G., White, S. D. M., \& Guiderdoni, B. 1993, MNRAS, 264, 201

Kennicutt, Jr., R. C. 1998, ApJ, 498, 541

Kereš, D., Katz, N., Weinberg, D. H., \& Davé, R. 2005, MNRAS, 363, 2

Khochfar, S., \& Silk, J. 2009, ApJ, 700, L21

Komatsu, E., \& Seljak, U. 2001, MNRAS, 327, 1353

Kravtsov, A. V., Gnedin, O. Y., \& Klypin, A. A. 2004, ApJ, 609, 482

Kritsuk, A. G., \& Norman, M. L. 2011 [arXiv: 1111.2827]

Labbé, I., González, V., Bouwens, R. J., et al. 2010, ApJ, 708, L26

Lada, C. J., Forbrich, J., Lombardi, M., \& Alves, J. F. 2012, ApJ, 745, 190

Lagos, C. D. P., Baugh, C. M., Lacey, C. G., et al. 2011, MNRAS, 418, 1649

Le Floc'h, E., Papovich, C., Dole, H., et al. 2005, ApJ, 632, 169

Le Floc'h, E., Aussel, H., Ilbert, O., et al. 2009, ApJ, 703, 222

Leauthaud, A., Finoguenov, A., Kneib, J.-P., et al. 2010, ApJ, 709, 97

Leauthaud, A., Tinker, J., Bundy, K., et al. 2012, ApJ, 744, 159

Lilly, S. J., Le Fevre, O., Hammer, F., \& Crampton, D. 1996, ApJ, 460, L1

Lu, Y., Kereš, D., Katz, N., et al. 2011, MNRAS, 416, 660

Madau, P., Ferguson, H. C., Dickinson, M. E., et al. 1996, MNRAS, 283, 1388 
Magnelli, B., Elbaz, D., Chary, R. R., et al. 2011, A\&A, 528, A35 Makino, N., Sasaki, S., \& Suto, Y. 1998, ApJ, 497, 555

Mandelbaum, R., Hirata, C. M., Broderick, T., Seljak, U., \& Brinkmann, J. 2006a, MNRAS, 370, 1008

Mandelbaum, R., Seljak, U., Cool, R. J., et al. 2006b, MNRAS, 372, 758

Martin, C. L. 1999, ApJ, 513, 156

McKay, T. A., Sheldon, E. S., Racusin, J., et al. 2001 [arXiv: astro-ph/0108013]

Mo, H. J., Mao, S., \& White, S. D. M. 1998, MNRAS, 295, 319

Monaco, P., Fontanot, F., \& Taffoni, G. 2007, MNRAS, 375, 1189

Moster, B. P., Somerville, R. S., Maulbetsch, C., et al. 2010, ApJ, 710, 903

Noeske, K. G., Weiner, B. J., Faber, S. M., et al. 2007, ApJ, 660, L43

Ogle, P., Boulanger, F., Guillard, P., et al. 2010, ApJ, 724, 1193

Okamoto, T., Gao, L., \& Theuns, T. 2008, MNRAS, 390, 920

Ostriker, J. P., Choi, E., Ciotti, L., Novak, G. S., \& Proga, D. 2010, ApJ, 722, 642

Peacock, J. A., Cole, S., Norberg, P., et al. 2001, Nature, 410, 169

Peebles, P. J. E. 1969, ApJ, 155, 393

Pfenniger, D., \& Combes, F. 1994, A\&A, 285, 94

Pfenniger, D., Combes, F., \& Martinet, L. 1994, A\&A, 285, 79

Planck Collaboration XVI. 2014, A\&A, 571, A16

Proga, D., Stone, J. M., \& Kallman, T. R. 2000, ApJ, 543, 686

Quinn, T., Katz, N., \& Efstathiou, G. 1996, MNRAS, 278, L49

Reddy, N. A., Steidel, C. C., Pettini, M., et al. 2008, ApJS, 175, 48

Reddy, N. A., Pettini, M., Steidel, C. C., et al. 2012, ApJ, 754, 25

Rees, M. J. 1986, MNRAS, 218, 25P

Rodighiero, G., Vaccari, M., Franceschini, A., et al. 2010, A\&A, 515, A8

Romeo, A. B., Burkert, A., \& Agertz, O. 2010, MNRAS, 407, 1223

Rubin, K. H. R., Prochaska, J. X., Ménard, B., et al. 2011, ApJ, 728, 55

Sargent, M. T., Béthermin, M., Daddi, E., \& Elbaz, D. 2012, ApJ, 747, L31

Shapiro, P. R., Giroux, M. L., \& Babul, A. 1994, ApJ, 427, 25
Shi, Y., Helou, G., Yan, L., et al. 2011, ApJ, 733, 87

Silk, J. 2003, MNRAS, 343, 249

Smit, R., Bouwens, R. J., Franx, M., et al. 2012, ApJ, 756, 14

Somerville, R. S. 2002, ApJ, 572, L23

Somerville, R. S., Hopkins, P. F., Cox, T. J., Robertson, B. E., \& Hernquist, L. 2008, MNRAS, 391, 481

Somerville, R. S., Gilmore, R. C., Primack, J. R., \& Domínguez, A. 2012, MNRAS, 423, 1992

Spergel, D. N., Verde, L., Peiris, H. V., et al. 2003, ApJS, 148, 175

Stark, D. P., Swinbank, A. M., Ellis, R. S., et al. 2008, Nature, 455, 775

Stark, D. P., Ellis, R. S., Bunker, A., et al. 2009, ApJ, 697, 1493

Stoll, R., Mathur, S., Krongold, Y., \& Nicastro, F. 2009 [arXiv: 0903 . 5310]

Sturm, E., González-Alfonso, E., Veilleux, S., et al. 2011, ApJ, 733, L16

Sutherland, R. S., \& Dopita, M. A. 1993, ApJS, 88, 253

Suto, Y., Sasaki, S., \& Makino, N. 1998, ApJ, 509, 544

Thoul, A. A., \& Weinberg, D. H. 1996, ApJ, 465, 608

Toomre, A. 1963, ApJ, 138, 385

Toomre, A. 1964, ApJ, 139, 1217

Tweed, D., Devriendt, J., Blaizot, J., Colombi, S., \& Slyz, A. 2009, A\&A, 506, 647

van de Voort, F., Schaye, J., Booth, C. M., Haas, M. R., \& Dalla Vecchia, C. 2011, MNRAS, 414, 2458

van den Bergh, S. 1996, AJ, 112, 2634

Veilleux, S., Cecil, G., \& Bland-Hawthorn, J. 2005, ARA\&A, 43, 769

Weinmann, S. M., Pasquali, A., Oppenheimer, B. D., et al. 2012, MNRAS, 426, 2797

White, S. D. M., \& Frenk, C. S. 1991, ApJ, 379, 52

Wilkins, S. M., Trentham, N., \& Hopkins, A. M. 2008, MNRAS, 385, 687

Yang, X., Mo, H. J., \& van den Bosch, F. C. 2009, ApJ, 695, 900

Zwaan, M. A., Meyer, M. J., Staveley-Smith, L., \& Webster, R. L. 2005, MNRAS, 359, L30 